\title{
Multiyear On-orbit Calibration and Performance of Terra MODIS Thermal Emissive Bands
}

\author{
Xiaoxiong Xiong ${ }^{\mathrm{a}}$, Kwo-Fu Chiang ${ }^{\mathrm{b}}$, Aisheng Wu $\mathrm{W}^{\mathrm{b}}$, William Barnes ${ }^{\mathrm{c}}$, Bruce Guenther ${ }^{\mathrm{c}}$, \\ and Vincent Salomonson ${ }^{\mathrm{d}}$ \\ ${ }^{a}$ Sciences and Exploration Directorate, NASA/GSFC, Greenbelt, MD 20771; \\ bscience Systems and Applications, Inc., 10210 Greenbelt Road, Lanham, MD 20706; \\ ${ }^{\mathrm{c}}$ University of Maryland, Baltimore County, 1000 Hilltop Circle, Baltimore, MD 21250; \\ ${ }^{\mathrm{d}}$ University of Utah, Salt Lake City, UT 84112.
}

\begin{abstract}
Since launch in December 1999, Terra MODIS has been making continuous Earth observations for more than seven years. It has produced a broad range of land, ocean, and atmospheric science data products for improvements in studies of global climate and environmental change. Among its 36 spectral bands, there are 20 reflective solar bands (RSB) and 16 thermal emissive bands (TEB). MODIS thermal emissive bands cover the mid-wave infrared (MWIR) and long-wave infrared (LWIR) spectral regions with wavelengths from 3.7 to $14.4 \mu \mathrm{m}$. They are calibrated on-orbit using an on-board blackbody (BB) with its temperature measured by a set of thermistors on a scan-by-scan basis. This paper will provide a brief overview of MODIS TEB calibration and characterization methodologies and illustrate on-board BB functions and TEB performance over more than seven years of on-orbit operation and calibration. Discussions will be focused on TEB detector short-term stability and noise characterization, and changes in long-term response (or system gain). Results show that Terra MODIS BB operation has been extremely stable since launch. When operated at its nominal controlled temperature of $290 \mathrm{~K}$, the BB temperature variation is typically less than $\pm 0.30 \mathrm{mK}$ on a scan-by-scan basis and there has been no time-dependent temperature drift. In addition to excellent short-term stability, most TEB detectors continue to meet or exceed their specified noise characterization requirements, thus enabling calibration accuracy and science data product quality to be maintained. Excluding the noisy detectors identified pre-launch and those that occurred post-launch, the changes in TEB responses have been less than $0.7 \%$ on an annual basis. The optical leak corrections applied to bands 32-36 have been effective and stable over the entire mission.
\end{abstract}

Keywords: Terra, MODIS, radiometer, thermal emissive bands, calibration, blackbody 


\section{Multiyear On-orbit Calibration and Performance of Terra MODIS Thermal Emissive Bands}

Xiaoxiong Xiong (1)

Kwo-Fu Chiang, Aisheng Wu (2)

William Barnes, Bruce Guenther (3)

Vincent Salomonson (4)

(1) 614.4, NASA/GSFC, Greenbelt, MD 20771

(2) Science Systems and Applications, Lanham, MD 20706

(3) UMBC, Baltimore, MD 21250

(4) U. of Utah, Salt Lake City, UT 84112

The Moderate Resolution Imaging Spectroradiometer (MODIS) on-board the NASA Earth Observing System (EOS) Terra spacecraft has successfully operated over 7 years since its launch in December 1999. The MODIS was designed to extend and enhance data records from a number of heritage sensors with improvements made in terms of spectral coverage, spatial resolutions, and global coverage. It makes observations in 36 spectral bands: 20 reflective solar bands (RSB) and 16 thermal emissive bands (TEB) with wavelengths from visible to long-wave infrared (LWIR), and at three spatial resolutions (nadir): $250 \mathrm{~m}$ ( 2 bands), $500 \mathrm{~m}$ ( 5 bands), and 1.0km ( 29 bands). For more than 7 years, MODIS data products have been extensively used by scientists worldwide for studies of the Earth's system of land, oceans, and atmosphere.

Accurate global observations from space by Earth-observing sensors provide useful and critical information to monitor long-term climate change and to address associated environmental issues. This, to a large extent, depends on the ability to accurately calibrate and characterize the sensors, from pre-launch to post-launch. An overview of MODIS calibration and characterization has been provided in a paper published in 2003 (Xiong et al.). The current paper focuses on Terra MODIS TEB on-orbit calibration and performance, including detector noise characterization, short-term stability, and longterm response changes, using more than 7 years of data from the on-board blackbody (BB) and space view source.

Results show that the on-board BB operation has been extremely effective and stable since launch, with temperature variation typically less than $\pm 0.30 \mathrm{mK}$ and no timedependent temperature drift when it is operated at its nominal controlled temperature of $290 \mathrm{~K}$. In addition to excellent short-term stability, most TEB detectors continue to meet or exceed the specified noise characterization requirements. The long-term response changes, less than $0.7 \%$ per year, are corrected with reference to the BB calibration source, thus enabling calibration accuracy and science data product quality to be continuously maintained. Challenging issues and lessons discussed in this paper will provide valuable information for future missions, such as the National Polar-orbiting Operational Environmental Satellite System (NPOESS) Visible/Infrared Imager/Radiometer Suite (VIIRS) and the Geostationary Operational Environmental Satellite Series-R (GOES-R) Advanced Baseline Imager (ABI). 


\section{Multiyear On-orbit Calibration and Performance of Terra MODIS Thermal Emissive Bands}

Xiaoxiong Xiong (1), Kwo-Fu Chiang (2), Aisheng Wu (2), William Barnes (3), Bruce Guenther (3), Vincent Salomonson (4)

(1) 614.4, NASA/GSFC, (2) MCST/SSAI, (3) UMBC, (4) U. of Utah

\section{Key Findings:}

Terra MODIS thermal emissive bands (TEB), with wavelengths from mid-wave infrared (MWIR) to long-wave infrared (LWIR), have been well calibrated and characterized since the sensor launch in December 1999, enabling many high-quality data products, such as land surface temperatures (LST) and sea surface temperatures (SST), to be continuously produced for science applications.

Its on-board blackbody (BB), the calibration source for the TEB with a total of 160 detectors, has been extremely stable with temperature variation typically less than $\pm 0.30 \mathrm{mK}$ at its nominal operating temperature. Most TEB detectors continue to meet or exceed their specified noise characterization requirements.

On-orbit characterization of TEB response versus scan angle (RVS), using data from spacecraft deep space maneuvers, has been successful with noticeable improvements made for the LWIR bands at large angle of incidences (AOI). In addition, the LWIR optical leak, identified pre-launch, has been significantly reduced using correction coefficients derived from on-orbit lunar observations.

Comprehensive and stringent pre-launch calibration and characterization activities and constant post-launch calibration efforts are critical for sensor on-orbit success to provide reliable data products of high quality and consistency. 


\title{
Multiyear On-orbit Calibration and Performance of Terra MODIS Thermal Emissive Bands
}

\author{
Xiaoxiong Xiong ${ }^{\mathrm{a}}$, Kwo-Fu Chiang ${ }^{\mathrm{b}}$, Aisheng $\mathrm{Wu}^{\mathrm{b}}$, William Barnes ${ }^{\mathrm{c}}$, Bruce Guenther ${ }^{\mathrm{c}}$, and \\ Vincent Salomonson ${ }^{\mathrm{d}}$ \\ ${ }^{a}$ Sciences and Exploration Directorate, NASA/GSFC, Greenbelt, MD 20771; \\ ${ }^{\mathrm{b}}$ Science Systems and Applications, Inc., 10210 Greenbelt Road, Lanham, MD 20706; \\ ${ }^{c}$ University of Maryland, Baltimore County, 1000 Hilltop Circle, Baltimore, MD 21250; \\ ${ }^{\mathrm{d}}$ University of Utah, Salt Lake City, UT 84112.
}

\begin{abstract}
Since launch in December 1999, Terra MODIS has been making continuous Earth observations for more than seven years. It has produced a broad range of land, ocean, and atmospheric science data products for improvements in studies of global climate and environmental change. Among its 36 spectral bands, there are 20 reflective solar bands (RSB) and 16 thermal emissive bands (TEB). MODIS thermal emissive bands cover the mid-wave infrared (MWIR) and long-wave infrared (LWIR) spectral regions with wavelengths from 3.7 to $14.4 \mu \mathrm{m}$. They are calibrated onorbit using an on-board blackbody (BB) with its temperature measured by a set of thermistors on a scan-by-scan basis. This paper will provide a brief overview of MODIS TEB calibration and characterization methodologies and illustrate on-board BB functions and TEB performance over more than seven years of on-orbit operation and calibration. Discussions will be focused on TEB detector short-term stability and noise characterization, and changes in long-term response (or system gain). Results show that Terra MODIS BB operation has been extremely stable since launch. When operated at its nominal controlled temperature of $290 \mathrm{~K}$, the BB temperature variation is typically less than $\pm 0.30 \mathrm{mK}$ on a scan-by-scan basis and there has been no timedependent temperature drift. In addition to excellent short-term stability, most TEB detectors continue to meet or exceed their specified noise characterization requirements, thus enabling calibration accuracy and science data product quality to be maintained. Excluding the noisy detectors identified pre-launch and those that occurred post-launch, the changes in TEB responses have been less than $0.7 \%$ on an annual basis. The optical leak corrections applied to bands 32-36 have been effective and stable over the entire mission.
\end{abstract}

Keywords: Terra, MODIS, radiometer, thermal emissive bands, calibration, blackbody 


\section{Introduction}

The Moderate Resolution Imaging Spectroradiometer (MODIS) Protoflight Model (PFM) was launched on December 18, 1999 on-board the NASA Earth Observing System (EOS) Terra spacecraft and, therefore, is referred to as the Terra MODIS. The Terra spacecraft is operated in a near sun-synchronous polar orbit at a nominal altitude of $705 \mathrm{~km}$ with an equatorial crossing time of 10:30AM (descending southwards). Among the five earth-observing sensors housed on the Terra spacecraft, MODIS is the most comprehensive sensor because of its design features and scientific applications. The MODIS was designed, based on inputs from many remote sensing scientists and a broad user community, in order to extend and enhance the data records from its heritage sensors, such as the AVHRR, CZCS, SeaWiFS, HIRS, and Landsat TM. Design improvements were made in terms of spectral coverage, spatial resolutions, and global coverage [1-2]. For more than seven years, Terra MODIS observations have been used extensively by scientists worldwide for studies of the Earth's system of land, oceans, and atmosphere [3-7]. The launch of the second MODIS instrument, Flight Model 1 (FM1), in May 2002 on-board the NASA EOS Aqua spacecraft has further enhanced its capability to monitor both short- and longterm climate and environmental changes through complementary morning (Terra) and afternoon (Aqua) observations [8]. Currently, there are approximately 40 science products that are routinely produced from both Terra and Aqua MODIS observations and distributed worldwide for various research studies and scientific applications.

MODIS collects data in 36 spectral bands with wavelengths in the visible (VIS), near infrared (NIR), short-wave infrared (SWIR), mid-wave infrared (MWIR), and long-wave infrared (LWIR). Observations are made over a scan angle range of $\pm 55^{\circ}$ relative to instrument nadir and at three nadir spatial resolutions: $250 \mathrm{~m}$ for bands $1-2,500 \mathrm{~m}$ for bands $3-7$, and $1.0 \mathrm{~km}$ for bands 8-36. MODIS bands 1-19 and 26 are the reflective solar bands (RSB), covering the VIS, NIR, and SWIR spectral regions. The remaining bands 20-25 and 27-36 are the thermal emissive bands (TEB), covering the MWIR and LWIR spectral regions. In order to maintain its stringent calibration and characterization requirements and to monitor its on-orbit performance, MODIS was built with a comprehensive set of onboard calibrators (OBC), including a solar diffuser (SD) and a solar diffuser stability monitor (SDSM) for RSB calibration, a blackbody (BB) for TEB calibration, and a spectro-radiometric calibration assembly (SRCA) for sensor spectral and spatial characterization. A space view (SV) port provides measurements of instrument thermal background and electronic offsets. Unlike the MODIS reflective solar bands, that only collect daytime reflected solar radiation, the thermal emissive bands make both daytime and nighttime measurements of the Earth view (EV) thermal emissions.

This paper focuses on Terra MODIS TEB calibration and performance. It provides a brief overview of the TEB calibration and characterization methodologies which use the on-board blackbody and space view source. The performance of the blackbody and the results of TEB detector noise characterization, short-term stability, and long-term response changes will be covered in the paper. Other topics briefly discussed in this paper include on-orbit characterization of Terra MODIS TEB response versus scan angle (RVS) and the optical leak corrections applied to the LWIR photoconductive (PC) bands 32-36. Existing challenging issues and lessons learned from Terra MODIS on-orbit operation and TEB calibration will be discussed 
at the end of the paper. Similar results for the Terra MODIS RSB have been reported elsewhere [9-10].

\section{Thermal Emissive Bands and On-board Blackbody}

\subsection{Thermal Emissive Bands (TEB)}

MODIS has 36 bands with a total of 490 individual detectors that are distributed according to their spectral wavelengths on four focal plane assemblies (FPAs): VIS, NIR, SMIR (for SWIR and MWIR) and LWIR. The MODIS spectral bands are aligned in the scan direction and linear detector arrays of each band are arranged in the track direction. There are 16 thermal emissive bands with wavelengths from 3.7 to $14.4 \mu \mathrm{m}$. Each band has 10 detectors, thus, there are a total of 160 TEB detectors. MODIS TEB bands/detectors are located on the two cold focal plane assemblies (CFPAs) as shown in Figure 1: the SMIR FPA for bands 20-25 and the LWIR FPA for band 27-36. Four SWIR bands (5-7 and 26) share the same FPA with the TEB MWIR bands 20-25. The MODIS reflective solar bands are located on the VIS and NIR FPAs. The MODIS TEB consist of photovoltaic (PV) HgCdTe detectors for bands 20-25 and 27-30 and photoconductive (PC) HgCdTe detectors for bands 31-36. The SMIR and LWIR FPAs are normally controlled at $83 \mathrm{~K}$ via a passive radiative cooler assembly and a micro-electric heater on each CFPA. Only one heater is used during nominal operation. When the sensor is operated with the A-side electronics, the heater on the LWIR FPA (primary heater) is used. The SMIR, or redundant, heater is used with the B-side electronics.

MODIS TEB observations are made at $1 \mathrm{~km}$ nadir spatial resolution and are primarily used for studies of surface/cloud/atmospheric temperature, water vapor, and cloud top altitude. Table 1 is a summary of design specifications for the thermal emissive bands, including their spectral wavelengths, bandwidths, typical scene radiances/temperatures (Ltyp/Ttyp), and noise equivalent radiance/temperature differences (NEdL/NEdT) at the specified typical scene radiances/temperatures. Calibration uncertainty requirements and primary applications of MODIS TEB are also listed in Table 1.

\subsection{On-board Blackbody (BB) and Space View (SV) Port}

The on-board blackbody and instrument space view port comprise a calibration system for the MODIS TEB. The BB shown in Figure 2 is a v-grooved panel $21 \mathrm{~cm}$ wide by $36 \mathrm{~cm}$ long with emissivity near 1.0 when it is viewed by the MODIS scan mirror. The BB temperature is measured by a set of 12 thermistors that are uniformly embedded beneath the BB front surface and traceable to the temperature standards of the National Institute of Standards and Technology (NIST). In the TEB calibration algorithm, an initial BB average temperature and its standard deviation $\left(\sigma_{\mathrm{T}}\right)$ are calculated each scan from all 12 thermistors. If the difference between an individual thermistor's temperature and the initial average temperature is outside of three times the standard deviation $\left(3 \sigma_{\mathrm{T}}\right)$, this thermistor will be excluded from being used to compute the final BB temperature average. This outlier rejection is implemented to reduce any potential impact due to unexpected large random noise in the thermistors and their associated electronics, and therefore to assure high quality BB temperature measurements. The SV port is a hole in the MODIS scan cavity wall through one of the electronics modules. MODIS TEB calibration is performed on a scan-by-scan basis with the BB serving as a calibration reference (or known 
spectral radiance) and the SV providing instrument thermal background. Each scan is 1.478 seconds in duration. A total of 50 calibration data samples are collected and averaged each scan from both the BB and SV sectors and used together to derive TEB on-orbit calibration coefficients.

\subsection{Terra MODIS TEB Pre-launch Calibration and Characterization}

Extensive pre-launch calibration and characterization activities were performed by the instrument vendor, Raytheon Santa Barbara Remote Sensing (SBRS) for the Terra MODIS TEB, including measurements to verify sensor spatial, spectral, and radiometric design specifications and to baseline its characteristics and performance [11-13]. Most TEB system level radiometric calibration measurements were made in a thermal vacuum (TV) environment. In order to evaluate sensor performance under different operating conditions, three instrument temperature plateaus, nominal $(273 \mathrm{~K})$, cold $(256 \mathrm{~K})$, and hot $(283 \mathrm{~K})$, were used during sensor TV calibration and characterization. A large aperture blackbody calibration source (BCS) served as the primary calibration source for the TEB radiometric calibration. The BCS is a buried first bounce design with an emissivity of better than 0.9995 over the MODIS TEB spectral range and temperature traceability to NIST standards.

At each instrument temperature plateau, a complete radiometric calibration was performed over a total of $21 \mathrm{BCS}$ temperatures ranging from $170 \mathrm{~K}$ to $340 \mathrm{~K}$. A space view source (SVS), similar to the BCS and operated at an extremely low temperature, was used to provide measurements of instrument background. MODIS TEB pre-launch radiometric calibration included studies of detector gains (or linear responses), dynamic range, nonlinearity, noise characterization, and short-term stability. A limited radiometric calibration, using 11 of the 21 BCS temperatures, was also performed at different CPFA temperatures $(77 \mathrm{~K}, 83 \mathrm{~K}$, and $85 \mathrm{~K})$. Additional measurements were made to characterize the difference between the sensor's primary and redundant electronic configurations. Key performance characteristics determined pre-launch included the BB emissivity and TEB detector nonlinear calibration coefficients. Both parameters are used in the on-orbit calibration algorithm.

\section{MODIS TEB On-orbit Calibration Algorithms}

\subsection{TEB On-orbit Calibration}

The MODIS TEB calibration is performed using a quadratic algorithm that relates the sensor's at-aperture radiance and its digital response (or digital number, $\mathrm{dn}$ ) with instrument background and electronic offset subtracted. This is a two-step process on a scan-by-scan basis applied to each band, detector, and mirror side. First a calibration coefficient $\left(b_{1}\right)$ is derived from each detector response to the $\mathrm{BB}$ calibration radiance and then the $\mathrm{EV}$ radiance is retrieved from the calibration coefficient and detector response to the EV. Considering the thermal emission contributions from the instrument scan cavity and scan mirror, the calibration and retrieval equations can be expressed by

and

$$
L_{C A L}=a_{0}+b_{1} \cdot d n_{B B}+a_{2} \cdot\left(d n_{B B}\right)^{2}
$$

$$
L_{R E T}=a_{0}+b_{1} \cdot d n_{E V}+a_{2} \cdot\left(d n_{E V}\right)^{2} .
$$


where the calibration radiance, $\mathrm{L}_{\mathrm{CAL}}$, is primarily from the $\mathrm{BB}$ thermal emission, plus small contributions from the scan mirror and instrument scan cavity, and the retrieving radiance, $\mathrm{L}_{\mathrm{RET}}$, includes a dominant EV radiance term and a small scan mirror term that varies with the EV angle of incidence (AOI) to the scan mirror [13-15]. The offset and nonlinear terms, $\mathrm{a}_{0}$ and $\mathrm{a}_{2}$, in the quadratic expression were initially derived from pre-launch calibration and are updated on-orbit using data collected from the on-board BB warm-up and cool-down processes. Note that the dominant linear term is denoted using $b_{1}$, instead of $a_{1}$, to emphasize its on-orbit computation on a scan-by-scan basis. The $\mathrm{dn}_{\mathrm{BB}}$ and $\mathrm{dn}_{\mathrm{EV}}$ are detector responses to the calibration and retrieving radiances (in digital numbers), $\mathrm{L}_{\mathrm{CAL}}$ and $\mathrm{L}_{\mathrm{RET}}$. Detector responses from $50 \mathrm{BB}$ data samples and that from $50 \mathrm{SV}$ data samples in each scan are averaged and used in the calibration. Thus,

and

$$
\left.d n_{B B}=\left\langle D N_{B B}\right\rangle_{A V G}-<D N_{S V}\right\rangle_{A V G},
$$

$$
d n_{E V}=D N_{E V}-<D N_{S V}>_{A V G}
$$

The EV radiance is retrieved for each EV digital number over the entire $\pm 55^{\circ} \mathrm{scan}$, a total of 1354 data samples (or frames) for each TEB detector. The BB, instrument scan cavity, and scan mirror temperatures are measured on-orbit and stored in the sensor telemetry packets. Their corresponding radiances are computed using the Planck equation weighted over each detector's relative spectral response (RSR). The RSR was determined from pre-launch spectral characterization. The EV scene brightness temperature can also be computed using the Planck equation from the retrieved EV spectral radiance.

\subsection{Special Considerations}

MODIS band 21 is designed for fire detection with a low gain setting and, consequently, a large temperature range. In order to reduce potential impacts on the scan-by-scan calibration due to variations in the detectors' relatively small responses (in digital numbers) to the BB, the band 21 linear coefficients are provided through look-up tables (LUTs) with their values derived from offline data analysis. In addition, the offset and nonlinear terms in the quadratic calibration algorithm are set to 0 because of their negligible impacts. This approach is currently used for both Terra and Aqua MODIS band 21 on-orbit calibration.

As previously described, bands 31-36 consist of photoconductive (PC) $\mathrm{HgCdTe}$ detectors that are located on the LWIR CFPA. From Terra MODIS pre-launch characterization, an optical leak from band 31 to the other PC bands (32-36) was identified. This problem has also been verified on-orbit by observing Earth view images and from MODIS lunar observations. In order to remove the PC optical leak impact on bands 32-36 on-orbit calibration and radiance retrieval, a linear correction algorithm was developed and implemented in the Terra MODIS Level 1B (L1B) algorithm. Because of the optical leak, the responses (dn) of bands 32-36 need to be corrected before being used in the calibration and radiance processes. This correction is applied to both the $\mathrm{BB}$ and $\mathrm{EV}$ responses on a detector-by-detector basis. The corrected detector responses for these bands, $\left(\mathrm{dn}_{\mathrm{BB}}\right)_{\mathrm{B} 32-36 \_C O R R}$ and $\left(\mathrm{dn}_{\mathrm{EV}}\right)_{\mathrm{B} 32-36 \_C O R R}$, are given by

and

$$
\left(d n_{B B}\right)_{B 32-36 \_C O R R}=\left(d n_{B B}\right)_{B 32-36}-C_{B 32-36} \cdot\left(d n_{B B}\right)_{B 31},
$$




$$
\left(d n_{E V}\right)_{B 32-36_{-} C O R R}=\left(d n_{E V}\right)_{B 32-36}-C_{B 32-36} \cdot\left(d n_{E V}\left[F O_{B 32-36}\right]\right)_{B 31} .
$$

The frame-offset $\left(\mathrm{FO}_{\mathrm{B} 32-36}\right)$ in the $\mathrm{EV}$ response (Eqn. 6) of band 31 is related to the relative locations of bands 32-36 to band 31 as shown in Figure 1. It is needed in order to capture instantaneous scene variations from the EV. There is no need to use the frame-offset term in the $\mathrm{BB}$ response as all data samples are collected from a uniform $\mathrm{BB}$ calibration source. The correction coefficients for bands 32-36, $\mathrm{C}_{\mathrm{B} 32-36}$, are derived from on-orbit lunar observations and verified from the EV measurements [16]. Because of lessons learned from Terra MODIS PC optical characterization and improvements made prior to Aqua MODIS launch, there is no PC optical leak in Aqua MODIS TEB [17].

\section{On-orbit Performance}

\subsection{TEB On-orbit Calibration and Characterization Activities}

Since launch, Terra MODIS has been operated in three different configurations and has experienced several spacecraft and sensor anomalies and events. As planned, it was operated atlaunch using its primary electronic configuration (A-side) and was then switched to the B-side (redundant electronics) on October 31, 2000 where it remained until June 15, 2001 when the Bside power supply was shut shown and unable to be re-activated. Consequently the A-side electronics configuration was brought back to resume Terra MODIS operation. Since September 17, 2002, Terra MODIS has been operated using the A-side electronics with the B-side formatter. This is often referred as the A1, B, A2, and A/B configuration sequence for Terra MODIS. The replacement of the A-side formatter with the B-side formatter was made to avoid "undesirable" error messages during sensor operation, even though these messages had no impact on the calibration and EV observations. Table 2 provides a timeline of all major configurations and events. In addition a number of different SMIR focal plane voltage biases, called Vdet and Itwk, were used during the first few months in an attempt to characterize and eliminate electronic crosstalk in the SWIR bands. Although some of the bias settings did reduce the electronic crosstalk, the reduction was accompanied by an increased number of noisy and inoperable detectors on the SMIR FPA. Consequently, no changes were made to the pre-launch designed Vdet/Itwk settings for both the A-side and B-side electronics.

For Terra MODIS, the BB temperature is set at $290 \mathrm{~K}$ for its nominal operation. Periodically a BB warm-up and cool-down (BB WUCD) operation is executed during which the BB temperature varies from instrument ambient of about $270 \mathrm{~K}$ to $315 \mathrm{~K}$ via electrical heating. This activity provides an opportunity to re-evaluate the quadratic calibration coefficients and allows the TEB detector responses to be examined on-orbit at a range of input radiances. Table 3 lists all the full BB WUCD activities implemented during Terra MODIS seven years of on-orbit operation. The BB WUCD was performed on nearly a weekly basis during the first year. Some of the BB WUCDs performed at the beginning of the mission (activities 9 to 33, omitted from Table 3) were limited operations designed to support the SWIR cross-talk investigations at different Vdet/Itwk settings for the SMIR FPA. After the first year, the BB WUCD has been performed on a quarterly basis.

\subsection{On-board Blackbody (BB) Performance}


The on-board BB temperature is set above the instrument ambient temperature at 290K during nominal on-orbit operation using a feedback controlled electrical heater embedded on the back of the BB. The BB temperature is measured by a set of 12 platinum resistance thermistors (PRT) on a scan-by-scan basis. These thermistors were calibrated pre-launch to the NIST temperature standard. The BB thermistor readouts are saved in each telemetry data packet using 12-bit digital numbers (DN) and then converted into temperature scales through a set of equations and associated coefficients in the L1B calibration process. To date, all 12 thermistors have continued to function satisfactorily. Table 4 is a summary of $B B$ temperature $\left(T_{B B}\right)$ stability from each individual thermistor at all major operational configurations (A1, B, A2, and A/B) listed in Table 2. The results, expressed in terms of BB temperature standard deviations, are derived using data over a complete orbit (about 100min). On a scan-by-scan basis the BB temperature variation has been less than $\pm 30 \mathrm{mK}$ for each of the 12 thermistors at all on-orbit operational configurations. The BB temperature used in the calibration is the average from all 12 thermistors. As mentioned in section 2, when computing the $\mathrm{BB}$ average temperature a 3-sigma exclusion algorithm has been applied in the L1B in order to remove any potential outliers or unexpected noise among the 12 thermistors.

The BB temperature long-term stability is illustrated in Figure 3 in which each data point is the average over a 5-min granule. Figure 3 a shows the BB temperature stability from an individual thermistor (number 1 ) and Figure $3 \mathrm{~b}$ from the average of all 12 thermistors. Some of the major instrument configuration changes and events described in Table 2 are marked using the vertical dotted lines. It is clear that when the instrument is under the same A-side operating configuration, the average $\mathrm{BB}$ temperature drift has been less than $2 \mathrm{mK}$ per year. There is also a small but noticeable BB temperature difference during B-side configuration ( $20 \mathrm{mK})$. Obviously this is not related to temperature drifting or stability. Instead, it is due to the BB temperature measuring electronics differences between the A-side and B-side configurations. Other small temperature fluctuations are primarily due to instrument operational conditions, such as nighttime to daytime and orbit-to-orbit differences, and seasonal and annual variations.

\subsection{Cold Focal Plane Assemblies Performance}

Since launch the temperatures of the Terra MODIS CFPA (SMIR and LWIR) have been nominally controlled at $83 \mathrm{~K}$ using a three-stage passive radiative cooler assembly. Figure 4 depicts the CFPA temperature long-term stability with Figure 4a for the SMIR FPA and Figure $4 \mathrm{~b}$ for the LWIR FPA temperature. The CFPA overall performance has been satisfactory for more than seven years of on-orbit operation, except for a short period at the beginning of the mission (see Table 2) when the radiative cooler lost its cooling capacity and thus the CFPAs' temperature stability. Following a sensor outgas operation, the radiative cooler regained its capacity and has been working extremely well since. As pointed out, the temperatures of the CFPAs are controlled by an electric heater on the LWIR FPA if the sensor is operated using the A-side electronics. Otherwise, for the B-side electronics, the CFPA temperatures are controlled by the heater on the SMIR FPA. The small FPA temperature difference or offset between A-side and B-side operation is simply due to the difference between the two settings pre-defined in the flight software. This situation is similar to the BB temperature difference between A-side and Bside operation. 
Table 5 is a summary of SMIR and LWIR FPA short-term temperature stability in the same operational configurations (A1, B, A2, and A/B) used in Table 4. The results from A1 configuration, showing relatively large CFPA temperature variations (50-60mK over an entire orbit) was derived during the time period when the CFPAs lost temperature control. For nominal operations, either with the A-side or B-side electronics, the SMIR FPA temperature short-term stability has been better than $35 \mathrm{mK}$ while the LWIR FPA has been better than $10 \mathrm{mK}$.

\subsection{Detector Noise Characterization}

MODIS TEB noise characterization is measured in terms of detector noise equivalent temperature difference (NEdT). This quantity is one of the key contributors to the TEB calibration uncertainty [18-19]. It is directly related to detector performance and data quality. The requirements shown in Table 1 are specified at the typical scene temperatures (Ttyp). Normally the NEdT at Ttyp is determined from measurements at a number of constant BB temperatures. For on-orbit characterization, it is often necessary to make an interpolation or extrapolation when computing the NEdT at Ttyp and to compare it with the design specification. For Terra MODIS, pre-launch characterization had identified 10 of the 160 as noisy detectors, all in band 36. On-orbit the NEdT is tracked for each TEB detector on a daily basis from its response to the on-board BB at a constant temperature. More comprehensive NEdT characterization is made using measurements during BB WUCD (see Table 3). Figure 5 shows examples of TEB noise characterization at different temperatures during BB WUCD process for bands 20, 22, 31, and 32. All 10 detectors of each band have shown similar NEdT characteristics.

After more than 7 years of on-orbit operation, all 160 Terra MODIS TEB detectors are still operable. Only 22 of the 160 TEB detectors are now considered to be noisy, including the 10 in band 36 that occurred pre-launch. Table 6 is a summary of noisy detector history for the Terra MODIS TEB. It is worth pointing out that all noisy detectors are located on the LWIR FPA. The noisy detector information is provided in the L1B data product through quality assurance (QA) flags. The on-orbit performance of detector noise characterization is illustrated in Table 7 using the band-averaged NEdT. The noisy detectors are not considered in the band-averaged NEdT except for band 36. The results presented in Table 7 are normalized to the specified requirements in Table 1. As expected, they are all below 1.0 except for band 36. B21 results are not included in Table 7 due to its low gain setting that leads to very small detector responses to the on-board BB.

\subsection{TEB Short-term Stability}

MODIS TEB calibration uses a quadratic algorithm (Eqn. 1) with dominant linear coefficients $\left(b_{1}\right)$ computed on a scan-by-scan basis from detectors' response to the on-board BB spectral radiance. The TEB short-term stability is illustrated in Figure 6 for bands 20, 22, 31, and 32 using detector scan-by-scan linear coefficient $b_{1}$ (mirror side 1 , middle detector only) over an entire orbit of calibration data sets. Results from mirror side 2 are similar to that from mirror side 1. For these bands, the short-term stability has been within $\pm 0.1 \%$. For reference purposes, the on-board BB and the cold FPA temperatures over the same orbit are provided in Figures 7 and 8. Normally the changes of BB temperatures will not affect the calibration coefficients. High (or low) BB temperature leads to high (or low) calibration input radiance and, consequently, induces large (or small) detector responses. On the other hand, the stability of the calibration coefficients could change significantly with FPA temperature as TEB detector responses have a strong 
temperature dependency. In order to continuously maintain TEB calibration stability and data product quality, the CFPA temperature is controlled at $83 \mathrm{~K}$. Slowly varying FPA temperatures should have little impact on TEB calibration, as all calibration coefficients are updated every scan, except for band 21 .

Results in Figures 6-8 are derived from data when the sensor was operated on the A-side electronics. For comparison purposes, Figure 9-11 present similar results using calibration data sets during B-side electronics operation. The small but noticeable features in Figures 9 and 11 are primarily due to that fact that CFPA temperatures are controlled via the electrical heater on the LWIR FPA (or SMIR FPA) when the sensor is operated with A-side (or B-side) electronics. It is clear that excellent short-term stability has been achieved for Terra MODIS TEB with both A-side and B-side electronics.

\subsection{TEB Long-term Response Changes}

Using seven years of on-orbit calibration data, Figure 12 shows Terra MODIS TEB calibration coefficient $b_{1}$ (defined in Eqn. 1) trending for bands 20, 22, 31, and 32 (mirror side 1). The vertical dashed lines are used to indicate the major operational configurations and events listed in Table 2. As expected these events often lead to impacts on the sensor response and, possibly, on its performance and data product continuity or consistency even though the calibration algorithm is designed to capture the response changes. It is obvious from Figure 12 that there were response changes in all four spectral bands when the sensor operation went through the A1, B, $\mathrm{A} 2$, and $\mathrm{A} / \mathrm{B}$ configuration sequence. In addition noticeably larger LWIR response changes occurred at the beginning of the mission. These are primarily due to changes in the CFPA temperatures during the period when the radiative cooler lost its cooling capacity (see Figure 4). The SMIR PV bands are less sensitive to FPA temperature changes as compared to the LWIR PC bands.

Excluding the expected impacts due to spacecraft and sensor events and anomalies, and those due to FPA temperature variations at the beginning of the mission, the annually averaged changes of TEB responses are less than $0.2 \%$ for the MWIR PV bands (except for B21), less than $0.7 \%$ for LWIR PV bands, and less than $0.4 \%$ for LWIR PC bands. B21, with an uncertainty requirement of $10 \%$, shows fluctuations within $5 \%$ over time. Typically bands within each group exhibit a very similar response pattern.

\subsection{Special Issues}

In addition to calibration and characterization activities performed using its on-board calibrators, special efforts have been made to improve Terra MODIS TEB calibration and data product quality, including on-orbit characterization of TEB response versus scan angle and corrections for the PC bands optical leak from band 31 to bands 32-36. MODIS is a scanning radiometer using a two-sided paddle wheel scan mirror to collect data over a field of view (FOV) of $\pm 55^{\circ}$ from the instrument nadir, which corresponds to the AOI on the scan mirror from $10.5^{\circ}$ to $65.5^{\circ}$. The system level RVS plays a critical role in TEB calibration and retrieval as the sensor response varies with the scan angle. MODIS TEB on-orbit calibration is performed at a fixed viewing

angle to the on-board BB. The scene radiances, however, are retrieved from observations over a 
range of viewing angles. In addition to the dominant EV radiance term, the retrieving radiance in Eqn. 2 contains a small scan mirror term that varies with Earth view AOI.

Terra MODIS pre-launch calibration and characterization failed to generate valid data sets that could be used to derive a system level RVS for the TEB. Consequently the TEB RVS had to be characterized on-orbit using data collected during spacecraft pitch maneuvers with the sensor making measurements of deep space [20]. Because of this, the at-launch RVS for the Terra MODIS TEB had to be constructed from scan mirror witness sample reflectance measurements. Initial on-orbit improvement was made to the mirror side 2 RVS relative to mirror side 1 using TEB detector responses to the nadir aperture door (NAD) when it was closed. Currently the Terra MODIS L1B calibration algorithm uses the TEB RVS derived from spacecraft pitch maneuvers. During spacecraft maneuvers, the EV radiance contains only a small scan mirror term that varies with scan mirror scan angle. Therefore the TEB RVS of each mirror side can be independently derived from detector responses during spacecraft pitch maneuvers. Figure 13 shows Terra MODIS TEB pre-launch RVS (no mirror side dependency) and on-orbit derived RVS (mirror side 1 and 2). The results are normalized to the BB AOI. Noticeable improvements have been made for the LWIR bands at large AOI.

Terra MODIS pre-launch characterization also identified an optical leak from band 31 to bands 32-36. Figure 14 shows an example of Terra MODIS lunar view responses (dn) for band 31 (no optical leak) and 35 (with optical leak). The small secondary peak in band 35 is due the out-ofband optical leak coming from band 31 . The frame difference of in-band response (primary peak) and the out-of-band response (secondary peak) matches the relative locations of bands 31 and 35 on the LWIR FPA. The optical leak impacts bands 32 to 36 with correction coefficients (relative to band 31 ) varying from $1 \%$ to $5 \%$. Because of this a correction algorithm is applied to bands 32-36 (Eqns. 5 and 6) with correction coefficients derived from on-orbit lunar observations. Figure 15 illustrates an example of improvements made by the PC optical leak correction algorithm using band $35 \mathrm{EV} \mathrm{L1B}$ images before and after correction. For more than seven years, the PC optical leak features have not changed. The correction coefficients have been very stable [16].

\section{Lessons and Challenges}

There have been many lessons learned from Terra MODIS TEB calibration and characterization, pre-launch and on-orbit, and successfully applied to Aqua MODIS design and characterization and, of course, to some new missions, such as the National Polar-orbiting Operational Environmental Satellite System (NPOESS) Visible/Infrared Imager/Radiometer Suite (VIIRS) and the Geostationary Operational Environmental Satellite Series-R (GOES-R) Advanced Baseline Imager (ABI). The following are a few of general lessons on sensor calibration:

- Full pre-launch radiometric calibration must be performed at different instrument temperatures, focal plane temperatures, electronic temperatures (if it can be independently controlled), and at different combinations of sensor operational configurations.

- Characterization should include measurements with high-contrast scenes or targets. 
- On-board BB must have a temperature control capability allowing it to be operated at different temperatures.

- The TEB focal plane temperature must be fully controlled and, if possible, the temperature of the optics along the TEB optical path must also be controlled to have better thermal stability.

- Independent characterization of the on-board BB emissivity must be made in addition to its thermistor temperature traceability.

- Spacecraft maneuvers and lunar observations should be considered as part of an on-orbit operation plan that can provide additional important information for sensor calibration and characterization.

- Calibration source stability and traceability are critical for high quality measurements and uncertainty assessments.

- Continuous efforts on sensor calibration and characterization from pre-launch characterization to on-orbit operation are necessary for a better understanding of sensor performance.

- Accurate radiometry over a wide spectral range requires numerous well designed calibration techniques and devices (MODIS make solar reflective and thermal emissive observations using the same scan mirror, fore-optics and telescope).

The PC band optical leak issue was another key lesson learned from Terra MODIS TEB calibration and characterization. With improvements made using information derived from its predecessor, Aqua MODIS completely eliminated PC optical leak problems. This is illustrated in Figure 16 with Aqua MODIS lunar view responses for band 31 and 35 and by comparing similar responses of Terra MODIS in Figure 14.

MODIS band 21 at $3.95 \mu \mathrm{m}$ was designed for fire detection with a low gain setting. Its saturation temperature was estimated to be $500 \mathrm{~K}$. However, on-orbit observations showed the temperatures retrieved from various types of fires are far below the band 21 saturation temperature of $500 \mathrm{~K}$. This is because band 21 makes EV observations at $1 \mathrm{~km}$ spatial resolution (nadir). Detailed analysis indicates that the saturation temperature at $3.95 \mu \mathrm{m}$ spectral band could be reduced to below $425 \mathrm{~K}$ for future sensor design consideration [21]. The change will lead to improved measurement resolution and calibration accuracy.

On-orbit calibration challenges include tracking TEB RVS changes and examining on-board BB emissivity stability over time. These two parameters are critical for TEB calibration and data product quality and are extremely difficult to measure without specially designed calibration activities that often require special spacecraft operation. During more than seven years of onorbit operation, there have been an increasing number of noisy detectors in the LWIR PV bands that, undoubtedly, will continue to present challenges for their calibration, including changes that will need to be made in the level 1B algorithm. In order to correct for instrument effects and changes in system response and to continuously maintain the data product quality, extensive calibration efforts have been made by the MODIS Characterization Support Team (MCST) at NASA/GSFC. In addition many vicarious calibration efforts and activities (for both TEB and RSB) have made significant contributions to MODIS calibration [22-27] 


\section{Summary}

Terra MODIS has been acquiring continuous global data sets to extend and improve studies of the Earth's land, oceans, and atmosphere for more than 7 years. MODIS has 16 thermal emissive bands with a total of 160 detectors, located on the MWIR and LWIR FPAs that are nominally controlled at $83 \mathrm{~K}$ and calibrated using an on-board blackbody with its temperature set at $290 \mathrm{~K}$. Extensive on-orbit calibration and validation activities by the MCST and the science community using the on-board calibrators and vicarious calibration targets to constantly monitor instrument performance have resulted in a marked improvement in the L1B TEB products. Excluding a few noisy detectors (22 out of 160) that occurred mainly in the LWIR bands and considering the fact that the sensor is performing well beyond its 5-year design life, the Terra MODIS overall performance has been satisfactory. The CFPA temperature and the on-board BB temperature have been extremely stable, resulting in excellent short-term and long-term response $\left(b_{1}\right)$ stability of the TEB detectors and, thus, of the calibration coefficients. In addition the PC optical leak has been stable and its correction algorithm remains effective. For additional information on the MODIS instrument operation, its on-orbit calibration and characterization activities, the sensor's current status, and the L1B algorithm or code changes and their LUTs updates, please contact the MODIS Characterization Support Team (MCST) or visit its web page: http://www.mcst.ssai.biz/mcstweb/index.html.

\section{Acknowledgment}

The authors would like to thank members of the MODIS Characterization Support Team (MCST) and the SBRS MODIS instrument team for their technical assistance and contributions made during pre-launch calibration and characterization and over seven years of on-orbit Terra MODIS operation.

\section{References:}

1. W.L. Barnes and V.V. Salomonson, "MODIS: A global image spectroradiometer for the Earth Observing System," Critical Reviews of Optical Science and Technology, CR47, 285307, 1993.

2. W.L. Barnes, V.V. Salomonson, B. Guenther, and X. Xiong, "Development, Characterization, and Performance of the EOS MODIS Sensors," Proceedings of SPIE Earth Observing Systems VIII, 5151, 337-345, 2003.

3. C.O. Justice, E. Vermote, J.R.G. Townshend, R. Defries, D.P. Roy, D.K. Hall, V.V. Salomonson, J.L. Privette, G. Riggs, A. Strahler, W. Lucht, R.B. Myneni, P. Lewis, and M.J. Barnsley, "The Moderate Resolution Imaging Spectroradiometer (MODIS): Land Remote Sensing for Global Change Research,” IEEE Trans. Geosci. Remote Sensing, 36, 1228-1249, 1998.

4. W.E. Esaias, M.R. Abbott, I. Barton, O.W. Brown, J.W. Campbell, K.L. Carder, D.K. Clark, R.L. Evans, F.E. Hoge, H.R. Gordon, W.P. Balch, R. Letelier, and P.J. Minnett, "An Overview of MODIS Capabilities for Ocean Science Observations," IEEE Trans. Geosci. Remote Sensing, 36, 1250-1265, 1998. 
5. M.D. King, W.P. Menzel, Y.J. Kaufman, D. Tanre, B.C. Gao, S. Platnick, S.A. Ackerman, L.A. Remer, R. Pincus, and P.A. Hubanks, "Cloud and Aerosol Properties, Precipitable Water, and Profiles of Temperature and Water Vapor from MODIS," IEEE Trans. Geosci. Remote Sensing, 41, 442-458, 2003.

6. V.V. Salomonson, W.L. Barnes, X. Xiong, S. Kempler, and E. Masuoka, "An Overview of the Earth Observing System MODIS Instrument and Associated Data Systems Performance," Proceedings of IGARSS, 2002.

7. B. Guenther, X. Xiong, V.V. Salomonson, W.L. Barnes, and J. Young, "On-orbit Performance of the Earth Observing System (EOS) Moderate Resolution Imaging Spectroradiometer (MODIS) and the Attendant Level 1-B Data Product," Remote Sensing of the Environment, 83, 16-30, 2002.

8. C.L. Parkinson, "Aqua: An Earth-Observing Satellite Mission to Examine Water and Other Climate Variables,” IEEE Trans. Geosci. Remote Sensing, 41, 173-183, 2003.

9. X. Xiong, J. Sun, J. Esposito, B. Guenther, and W. L. Barnes, MODIS Reflective Solar Bands Calibration Algorithm and On-orbit Performance, Proceedings of SPIE - Optical Remote Sensing of the Atmosphere and Clouds III, 4891, 95-104, 2003.

10. X. Xiong, J. Sun, W. Barnes, V. Salomonson, J. Esposito, H. Erives, and B. Guenther, "Multi-year On-orbit Calibration and Performance of Terra MODIS Reflective Solar Bands," IEEE Transactions on Geosci and Remote Sensing, Vol. 45, No. 4, 879-889, 2007.

11. W.L. Barnes, T.S. Pagano, and V.V. Salomonson, "Prelaunch Characteristics of the Moderate Resolution Imaging Sectroradiometer (MODIS) on EOS-AM1," IEEE Trans. Geosci. Remote Sensing, 36, 1088-1100, 1998.

12. X. Xiong, K. Chiang, N. Chen, S. Xiong, W.L. Barnes, and B. Guenther, "Results and Lessons from MODIS Thermal Emissive Bands Calibration: Pre-launch to On-orbit," Proceedings of SPIE - Earth Observing Systems XI, Vol. 6296, 62960A, doi:10.1117/12.680992, 2006.

13. X. Xiong and W.L. Barnes, "An Overview of MODIS Radiometric Calibration and Characterization," Advances in Atmospheric Sciences, 23 (1), 69-79, 2006.

14. X. Xiong, K. Chiang, J. Esposito, B. Guenther, and W.L. Barnes, "MODIS On-orbit Calibration and Characterization," Metrologia, 40, 89-92, 2003.

15. X. Xiong, K. Chiang, B. Guenther, and W.L. Barnes, "MODIS Thermal Emissive Bands Calibration Algorithm and On-orbit Performance", Proceedings of SPIE-Optical Remote Sensing of the Atmosphere and Clouds III, 4891, 392-401, 2003.

16. W. Li, X. Xiong, K. Chiang, and G. Toller, "Evaluation of Terra MODIS PC Bands Optical Leak Correction Algorithm", Proceedings of SPIE - Earth Observing Systems X, Vol. 5882, 588219, doi:10.1117/12.614509, 2005.

17. X. Xiong, W.L. Barnes, B. Guenther, and R.E. Murphy, "Lessons Learned from MODIS Calibration and Characterization," J. of Advances in Space Research, 32/11, 2017-2122, 2003.

18. K. Chiang, X. Xiong, A. Wu, and W.L. Barnes, "MODIS Thermal Emissive Bands Calibration Uncertainty Analysis," Proceedings of SPIE - Earth Observing Systems IX, 5542, 437-447, 2004.

19. X. Xiong, J. Sun, A. Wu, K. Chiang, J. Esposite, and W.L. Barnes, "Terra and Aqua MODIS Calibration Algorithms and Uncertainty Analysis," Proceedings of SPIE - Sensors, Systems, and Next Generation of Satellites IX, 5978, 59780V, doi:10.1117/12.627637, 2005. 
20. X. Xiong, V.V. Salomonson, K. Chiang, A. Wu, B. Guenther, and W.L. Barnes, "On-orbit Characterization of RVS for MODIS Thermal Emissive Bands," Proceedings of SPIE Passive Optical Remote Sensing of the Atmosphere and Clouds IV, 5652, 210-218, 2005.

21. B. Gao, X. Xiong, R. Li, and D. Wang, "Evaluation of The MODIS Special 3.95- $\mu \mathrm{m}$ Fire Channel and Implications on Fire Channel Selections for Future Satellite Instrument," Journal of Applied Remote Sensing, 2007.

22. X. Xiong, A. Wu, and C. Gao, "On-Orbit Calibration and Inter-Comparison of Terra and Aqua MODIS Surface Temperature Spectral Bands," accepted for International Journal of Remote Sensing, 2007.

23. A. Wu, X. Xiong, C. Cao, F. Wu, and W.L. Barnes, "Inter-comparison of Radiometric Calibration of Terra and Aqua MODIS $11 \mu \mathrm{m}$ and $12 \mu \mathrm{m}$ Bands," Proceedings of SPIEEarth Observing Systems IX, 5542, 248-258, 2004.

24. Z. Wan, Y. Zhang, Q. Zhang, and Z. Li, "Quality Assessment and Validation of the MODIS Global Land Surface Temperature," Int. J. Remote Sensing, Vol 25, No 1, 261-274, 2004.

25. P. Minnett, O. Brown, R. Evans, E. Key, E. Kearns, K. Kilpatrick, A. Kumar, K. Maillet, and M. Szczodrak, "Sea-surface Temperature Measurements from the Moderate-Resolution Imaging Spectroradiometer (MODIS) on Aqua and Terra,, Proceedings of IGARSS 2004, 4576-4579, 2004.

26. S. Hook, R. Vaughan, H. Tonooka, and S. Schladow, "Absolute Radiometric In-Flight Validation of Mid and Thermal Infrared Data from ASTER and MODIS Using the Lake Tahoe CA/NV, USA Automated Validation Site," accepted for IEEE Trans. Geosci. Remote Sensing, 2007.

27. C. Moeller, S. Hook, D. Tobin, V. Walden, “Assessing MODIS LWIR band calibration accuracy," Proceedings of SPIE - Earth Observing Systems XI, Vol. 6296, 62960B, doi:10.1117/12.680993, 2006. 
TABLE 1 MODIS THERMAL EMISSIVE BAND SPECIFICATIONS

\begin{tabular}{cccccccccl}
\hline Band & CW & BW & Ltyp & Ttyp & NEdL & NEdT & UC (\%) & UC (T) & Primary Use \\
\hline 20 & 3.75 & 0.18 & 0.45 & 300 & 0.0010 & 0.05 & 0.75 & 0.18 & \\
21 & 3.96 & 0.06 & 2.38 & 335 & 0.0154 & 0.20 & 1 & 0.31 & Surface/Cloud \\
22 & 3.96 & 0.06 & 0.67 & 300 & 0.0019 & 0.07 & 1 & 0.25 & Temperature \\
23 & 4.05 & 0.06 & 0.79 & 300 & 0.0022 & 0.07 & 1 & 0.25 & \\
\hline 24 & 4.47 & 0.07 & 0.17 & 250 & 0.0022 & 0.25 & 1 & 0.19 & Atmospheric \\
25 & 4.52 & 0.07 & 0.59 & 275 & 0.0062 & 0.25 & 1 & 0.24 & Temperature \\
\hline 27 & 6.72 & 0.36 & 1.16 & 240 & 0.0108 & 0.25 & 1 & 0.27 & \\
28 & 7.33 & 0.30 & 2.19 & 250 & 0.0172 & 0.25 & 1 & 0.32 & Water Vapor \\
29 & 8.55 & 0.30 & 9.59 & 300 & 0.0090 & 0.05 & 1 & 0.53 & \\
\hline 30 & 9.73 & 0.30 & 3.70 & 250 & 0.0219 & 0.25 & 1 & 0.42 & Ozone \\
\hline 31 & 11.03 & 0.50 & 9.56 & 300 & 0.0070 & 0.05 & 0.5 & 0.34 & Surface/Cloud \\
32 & 12.02 & 0.50 & 8.95 & 300 & 0.0061 & 0.05 & 0.5 & 0.37 & Temperature \\
\hline 33 & 13.34 & 0.30 & 4.53 & 260 & 0.0183 & 0.25 & 1 & 0.62 & \\
34 & 13.64 & 0.30 & 3.77 & 250 & 0.0161 & 0.25 & 1 & 0.59 & Cloud Top Altitude \\
35 & 13.94 & 0.30 & 3.11 & 240 & 0.0141 & 0.25 & 1 & 0.55 & \\
36 & 14.24 & 0.30 & 2.08 & 220 & 0.0154 & 0.35 & 1 & 0.47 & \\
\hline
\end{tabular}

CW: Center Wavelengths in $\mu \mathrm{m}$;

BW: Bandwidths in $\mu \mathrm{m}$;

Ltyp: Typical Spectral Radiance in $\mathrm{W} / \mathrm{m}^{2} / \mu \mathrm{m} / \mathrm{sr}$;

Ttyp: Temperature at Ltyp in Kelvin (K);

NEdL: Noise Equivalent Radiance Difference in $\mathrm{W} / \mathrm{m}^{2} / \mu \mathrm{m} / \mathrm{sr}$;

NEdT: Noise Equivalent Temperature Difference in $\mathrm{K}$;

UC (\%): Uncertainty in \%;

UC (T): Uncertainty in Temperature.

TABLE 2 TERRA MODIS MAJOR OPERATIONAL CONFIGURATIONS AND EVENTS

\begin{tabular}{c|cl}
\hline & Y/ M/ D & \\
\hline & $1999 / 12 / 18$ & Terra launch \\
& $2000 / 02 / 13$ & Sensor operated in science mode using A-side electronics (default configuration setting) \\
A1 & $2000 / 02 / 24$ & Nadir door open (“First Light”) \\
& $2000 / 06 / 08$ & Cold FPA lost control (slowly became worse; fixed with an outgas process) \\
& $2000 / 08 / 05$ & Formatter anomaly; sensor to low power mode; outgas performed \\
& $2000 / 08 / 19$ & Return to science mode using A-side electronics \\
\hline \multirow{3}{*}{ B } & $2000 / 10 / 30$ & Switch to all B-side electronics \\
& $2001 / 06 / 15$ & Power supply (PS) 2 anomaly; sensor transitioned to the survival mode \\
\hline \multirow{3}{*}{ A2 } & $2001 / 07 / 02$ & Switch to all A-side electronics configuration \\
& $2002 / 03 / 19$ & Safe mode during spacecraft inclination maneuver \\
& $2002 / 03 / 27$ & Return to science mode; nadir door open on 2002087 \\
\hline \multirow{4}{*}{ A/B } & $2002 / 09 / 17$ & Switch to A-side electronics and B-side formatter \\
& $2003 / 12 / 16$ & Safe mode due to Attitude Control Electronics anomaly \\
& $2003 / 12 / 24$ & Return to science mode \\
& $2006 / 08 / 22$ & Nadir and SV doors accidentally closed; opened 2 hours later \\
\hline
\end{tabular}


TABLE 3 TERRA MODIS BB WARM-UP AND COOL-DOWN ACTIVITIES (ITWK/VDET IS FOR SMIR FPA VOLTAGE BIAS)

\begin{tabular}{ccc|ccc|ccc}
\hline No & Date & Itwk/Vdet & No & Date & Itwk/Vdet & No & Date & Itwk/Vdet \\
\hline 1 & 2000056 & $79 / 190$ & 38 & 2001002 & $79 / 110$ & 51 & 2004114 & $79 / 190$ \\
2 & 2000057 & $110 / 226$ & 39 & 2001103 & $79 / 110$ & 52 & 2004203 & $79 / 190$ \\
3 & 2000058 & $110 / 220$ & 40 & 2001194 & $79 / 190$ & 53 & 2004297 & $79 / 190$ \\
4 & 2000061 & $110 / 220$ & 41 & 2001291 & $79 / 190$ & 54 & 2005017 & $79 / 190$ \\
5 & 2000064 & $110 / 224$ & 42 & 2002018 & $79 / 190$ & 55 & 2005101 & $79 / 190$ \\
6 & 2000065 & $110 / 228$ & 43 & 2002110 & $79 / 190$ & 56 & 2005201 & $79 / 190$ \\
7 & 2000067 & $110 / 226$ & 44 & 2002206 & $79 / 190$ & 57 & 2005281 & $79 / 190$ \\
8 & 2000069 & $110 / 226$ & 45 & 2002293 & $79 / 190$ & 58 & 2006014 & $79 / 190$ \\
$\ldots$ & $\ldots$ & $\ldots$ & 46 & 2003034 & $79 / 190$ & 59 & 2006100 & $79 / 190$ \\
34 & 2000239 & $110 / 226$ & 47 & 2003114 & $79 / 190$ & 60 & 2006200 & $79 / 190$ \\
35 & 2000292 & $79 / 110$ & 48 & 2003204 & $79 / 190$ & 61 & 2006259 & $79 / 190$ \\
36 & 2000305 & $79 / 110$ & 49 & 2003302 & $79 / 190$ & 62 & 2006343 & $79 / 190$ \\
37 & 2000325 & $79 / 110$ & 50 & 2004007 & $79 / 190$ & 63 & 2007069 & $79 / 190$ \\
\hline
\end{tabular}

TABLE 4 SUMMARY OF BB TEMPERATURE $\left(\mathrm{T}_{\mathrm{BB}}\right)$ STABILITY

\begin{tabular}{|c|c|c|c|c|c|c|c|c|}
\hline \multirow{2}{*}{ Thermistor } & \multicolumn{2}{|c|}{ A1 (2000213) (K) } & \multicolumn{2}{c|}{ B (2001103)(K) } & \multicolumn{2}{c|}{ A2 (2002205) (K) } & \multicolumn{2}{c|}{ A/B (2007069) (K) } \\
\cline { 2 - 9 } & Avg & Sigma & Avg & Sigma & Avg & Sigma & Avg & Sigma \\
\hline 1 & 290.007 & 0.026 & 290.031 & 0.021 & 290.008 & 0.027 & 290.012 & 0.025 \\
2 & 289.974 & 0.026 & 290.008 & 0.019 & 289.979 & 0.028 & 290.002 & 0.022 \\
3 & 290.003 & 0.024 & 290.045 & 0.018 & 290.008 & 0.026 & 290.024 & 0.025 \\
4 & 289.982 & 0.025 & 290.026 & 0.019 & 289.986 & 0.028 & 290.008 & 0.023 \\
5 & 289.977 & 0.022 & 290.016 & 0.018 & 289.983 & 0.025 & 290.004 & 0.022 \\
6 & 289.972 & 0.022 & 289.990 & 0.028 & 289.977 & 0.026 & 289.989 & 0.024 \\
7 & 290.013 & 0.026 & 290.054 & 0.019 & 290.018 & 0.025 & 290.029 & 0.025 \\
8 & 290.002 & 0.027 & 290.040 & 0.021 & 290.005 & 0.029 & 290.011 & 0.027 \\
9 & 290.005 & 0.027 & 290.021 & 0.021 & 290.005 & 0.025 & 290.009 & 0.024 \\
10 & 289.997 & 0.026 & 290.010 & 0.023 & 289.999 & 0.027 & 290.000 & 0.023 \\
11 & 289.951 & 0.025 & 289.994 & 0.019 & 289.958 & 0.030 & 289.997 & 0.021 \\
12 & 289.953 & 0.027 & 290.000 & 0.016 & 289.959 & 0.028 & 289.998 & 0.025 \\
\hline
\end{tabular}

TABLE 5 SUMMARY OF CFPA TEMPERATURE $\left(\mathrm{T}_{\mathrm{BB}}\right)$ STABILITY

\begin{tabular}{|c|c|c|c|c|c|c|c|c|}
\hline \multirow{2}{*}{ FPA } & \multicolumn{2}{|c|}{ A1 (2000213) (K) } & \multicolumn{2}{c|}{ B (2001103) (K) } & \multicolumn{2}{c|}{ A2 (2002205) (K) } & \multicolumn{2}{c|}{ A/B (2007069) (K) } \\
\cline { 2 - 9 } & Avg & Sigma & Avg & Sigma & Avg & Sigma & Avg & Sigma \\
\hline SMIR & 84.430 & 0.066 & 83.200 & 0.028 & 83.321 & 0.032 & 83.375 & 0.034 \\
LWIR & 83.969 & 0.051 & 83.019 & 0.003 & 83.024 & 0.004 & 83.020 & 0.005 \\
\hline
\end{tabular}


TABLE 6 SUMMARY OF TERRA MODIS TEB NOISY DETECTOR HISTORY

\begin{tabular}{ccl}
\hline Time & Event & \multicolumn{1}{c}{ Band (Detector) } \\
\hline Pre-launch & & B36(all) \\
2000055.1527 & Nadir Door Open & B33(1), B34(7,8), B36(all) \\
2000160.0000 & CFPA Lost Control & B30(5) B33(1), B34(7,8), B36(all) \\
2000218.2210 & Formatter Anomaly & B27(6), B30(5), B33(1), B34(6,7,8), B36(all) \\
2000304.1420 & Switch to B-Side & B27(6), B30(5), B33(1), B34(6,7,8), B36(all) \\
2001019.1415 & N/A & B27(6), B30(5, 8), B33(1), B34(6,7,8), B36(all) \\
2001183.2245 & Switch to A-Side & B27(6), B30(5, 8), B33(1), B34(6,7,8), B36(all) \\
2002078.1615 & Safe Mode & B27(6), B28(3), B30(5,8), B33(1), B34(56,7,8), B36(all) \\
2003350.1305 & Safe Mode & B27(1,6), B28(8), B30(5,8), B33(1), B34(6,7,8), B36(all) \\
2005130.1345 & SAA (Day) & B27(1,6), B28(1,8), B29(6), B30(5,8), B33(1), B34(6,7,8), B36(all) \\
2005309.1510 & N/A & B27(1,6), B28(8,9), B29(6), B30(5,8), B33(1), B34(6,7,8), B36(all) \\
2006155.0210 & SAA (Night) & B27(1,6), B28(8,9), B29(6), B30(3,5,8), B33(1), B34(6,7,8), B36(all) \\
\hline
\end{tabular}

TABLE 7 TEB DETECTOR NOISE CHARACTERIZATION RESULTS (NORMALIZED TO SPECIFICATIONS AT LTYP)

\begin{tabular}{cccccccccccc}
\hline Band & 2000A & 2000B & 2001B & 2001A & 2002A & 2002AB & 2003AB & 2004AB & 2005AB & 2006AB & 2007AB \\
\hline 20 & 0.49 & 0.52 & 0.52 & 0.52 & 0.52 & 0.52 & 0.52 & 0.52 & 0.52 & 0.52 & 0.52 \\
22 & 0.39 & 0.39 & 0.38 & 0.39 & 0.39 & 0.39 & 0.39 & 0.39 & 0.39 & 0.39 & 0.39 \\
23 & 0.34 & 0.36 & 0.36 & 0.36 & 0.36 & 0.36 & 0.36 & 0.36 & 0.36 & 0.36 & 0.36 \\
24 & 0.36 & 0.38 & 0.41 & 0.38 & 0.38 & 0.38 & 0.37 & 0.37 & 0.36 & 0.37 & 0.36 \\
25 & 0.19 & 0.20 & 0.21 & 0.20 & 0.20 & 0.20 & 0.19 & 0.19 & 0.19 & 0.19 & 0.19 \\
27 & 0.28 & 0.36 & 0.53 & 0.29 & 0.30 & 0.29 & 0.30 & 0.35 & 0.36 & 0.36 & 0.34 \\
28 & 0.18 & 0.19 & 0.27 & 0.15 & 0.16 & 0.15 & 0.16 & 0.23 & 0.23 & 0.23 & 0.25 \\
29 & 0.64 & 0.66 & 0.69 & 0.66 & 0.66 & 0.67 & 0.66 & 0.67 & 0.67 & 0.68 & 0.67 \\
30 & 0.33 & 0.55 & 0.63 & 0.30 & 0.36 & 0.35 & 0.37 & 0.33 & 0.34 & 0.33 & 0.31 \\
31 & 0.64 & 0.61 & 0.57 & 0.61 & 0.62 & 0.61 & 0.61 & 0.61 & 0.61 & 0.61 & 0.60 \\
32 & 0.81 & 0.80 & 0.73 & 0.80 & 0.80 & 0.78 & 0.79 & 0.79 & 0.79 & 0.79 & 0.78 \\
33 & 0.55 & 0.58 & 0.53 & 0.51 & 0.52 & 0.51 & 0.51 & 0.52 & 0.52 & 0.52 & 0.52 \\
34 & 0.82 & 0.97 & 0.78 & 0.77 & 0.77 & 0.77 & 0.76 & 0.76 & 0.76 & 0.75 & 0.78 \\
35 & 0.97 & 0.90 & 0.89 & 0.90 & 0.93 & 0.88 & 0.88 & 0.90 & 0.91 & 0.91 & 0.89 \\
36 & 1.20 & 1.07 & 1.15 & 1.07 & 1.22 & 1.10 & 1.10 & 1.14 & 1.13 & 1.10 & 1.15 \\
\hline
\end{tabular}




\section{Figure Captions}

Figure 1. MODIS cold focal plane assemblies (CFPAs): short- and mid-wave infrared (SWIR/MWIR) FPA and long-wave infrared (LWIR) FPA

Figure 2. MODIS on-board blackbody (BB)

Figure 3a. On-board blackbody (BB) temperature long-term stability from thermistor 1

Figure 3b. On-board blackbody (BB) temperature long-term stability from average of all 12 thermistors

Figure 4a. SMIR focal plane assembly (FPA) temperature long-term stability

Figure 4b. LWIR focal plane assembly (FPA) temperature long-term stability

Figure 5. TEB detector noise characterization (NEdT) at different blackbody (BB) temperatures (data from July 20, 2006 BB warm-up) for bands 20, 22, 31, and 32

Figure 6. TEB responsè $\left(\mathrm{b}_{1}\right)$ short-term stability for bands 20, 22, 31, and 32 (middle detector) with A-side electronics (data from 2007069)

Figure 7. BB temperature (average of all 12 thermistors) short-term stability (same data as Figure 6)

Figure 8. CFPA (SMIR and LWIR) temperature short-term stability (same data as Figure 6)

Figure 9. TEB response ( $\left.b_{1}\right)$ short-term stability for bands 20, 22, 31, and 32 (middle detector) with B-side electronics (data from 2001002)

Figure 10. BB temperature (average of all 12 thermistors) short-term stability (same data as Figure 9)

Figure 11. CFPA (SMIR and LWIR) temperature short-term stability (same data as Figure 9)

Figure 12. TEB calibration coefficient $b_{1}\left(\mathrm{~W} / \mathrm{m}^{\wedge} 2 / \mathrm{sr} / \mu \mathrm{m} / \mathrm{dn}\right)$ long-term stability for bands 20 , 22,31 , and 32 (all detectors)

Figure 13. TEB response versus scan angle (RVS), normalized at blackbody angle of incidence (AOI): pre-launch (solid lines); on-orbit mirror side 1 (dashed lines); on-orbit mirror side 2 (dotted lines). (X-axis: AOI in degrees; Y-axis: Normalized RVS, unitless)

Figure 14. Terra MODIS lunar response (dn) from band 31 (no optical leak) and band 35 (with optical leak) 
Figure 15. Terra MODIS PC optical leak correction: (a) L1B image from band 31 (no optical leak); (b) L1B image from band 35 (with optical leak, no correction); (c) L1B image from band 35 (with optical leak, with correction)

Figure 16. Aqua MODIS lunar response (dn) from band 31 (no optical leak) and band 35 (no optical leak) 
Figure 1
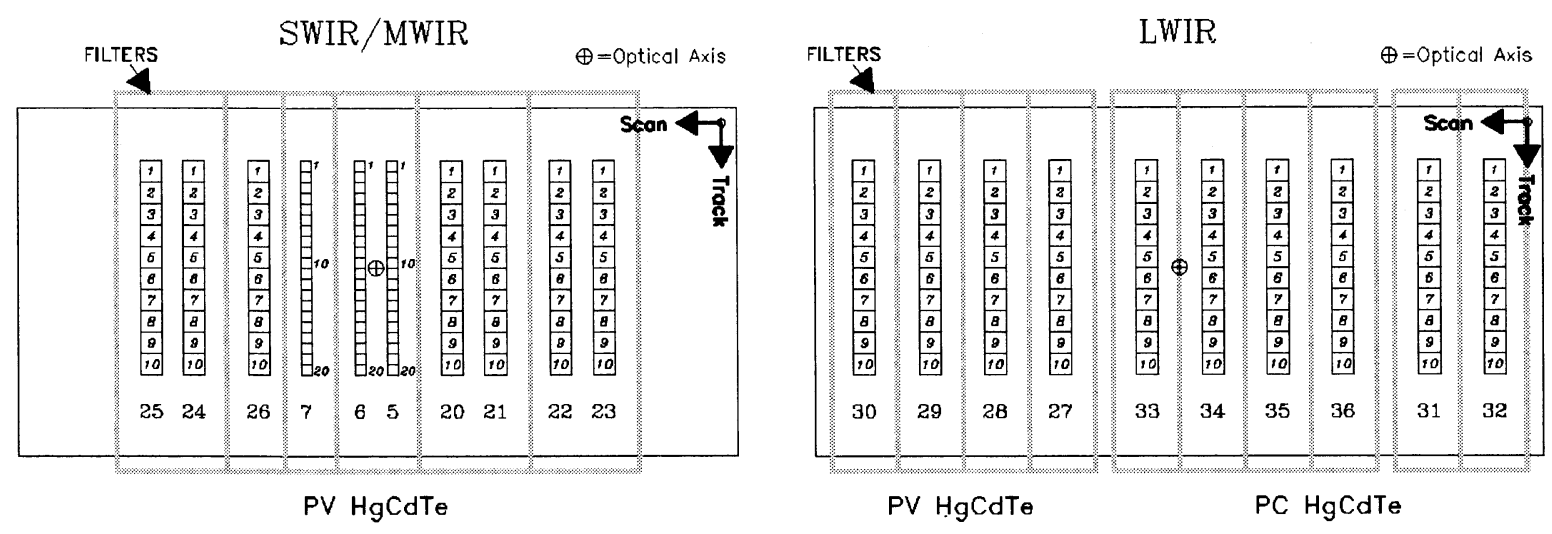

Figure 2

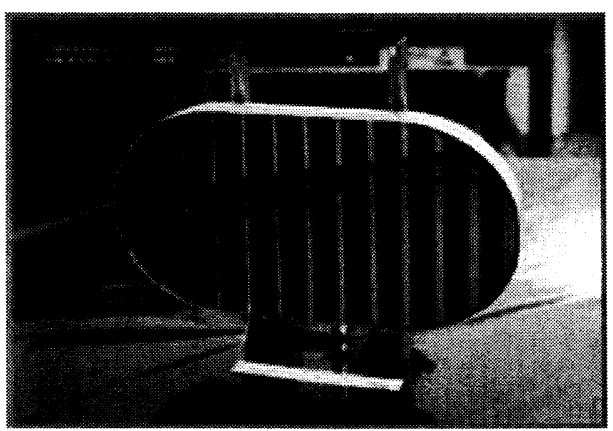


Figure 3a

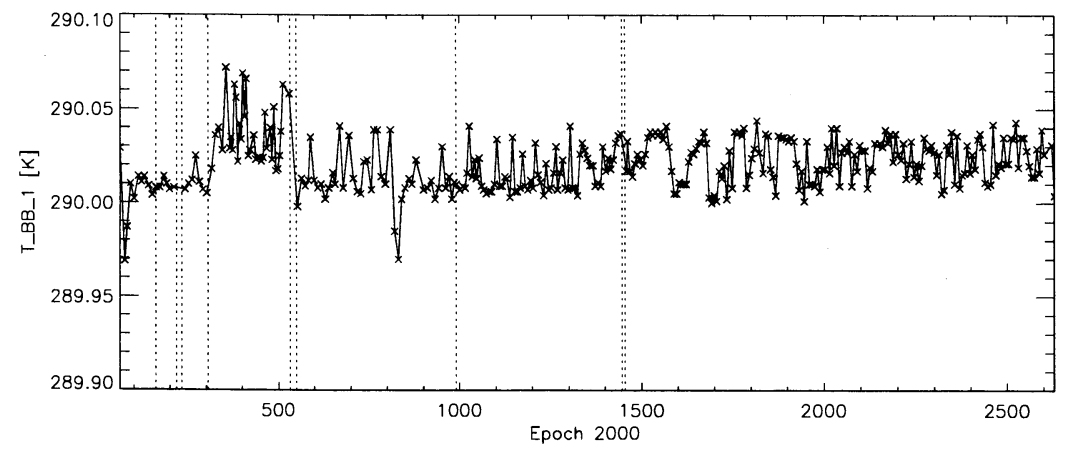

Figure $3 b$

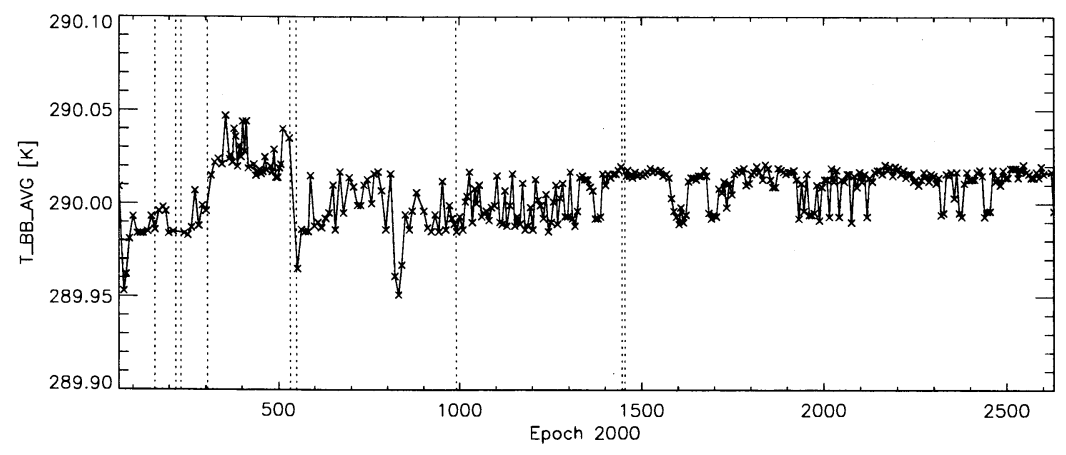

Figure 4a

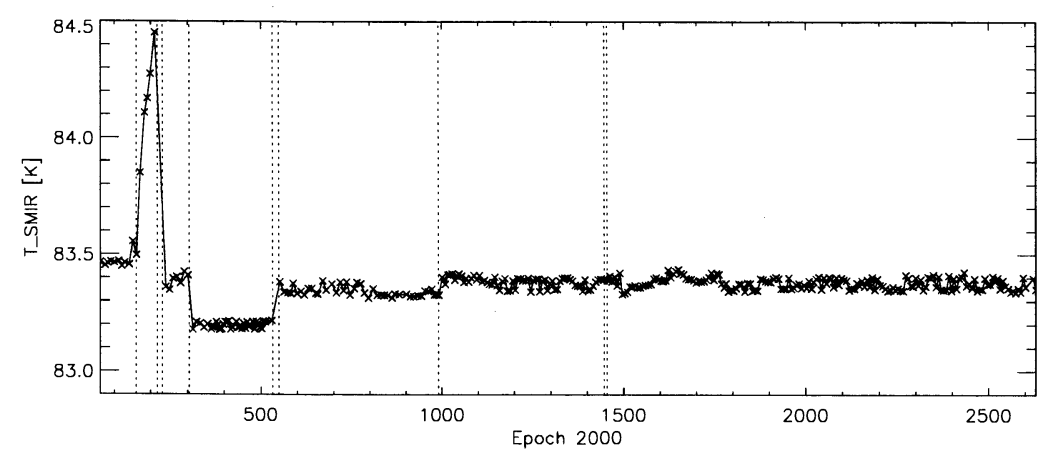

Figure 4b

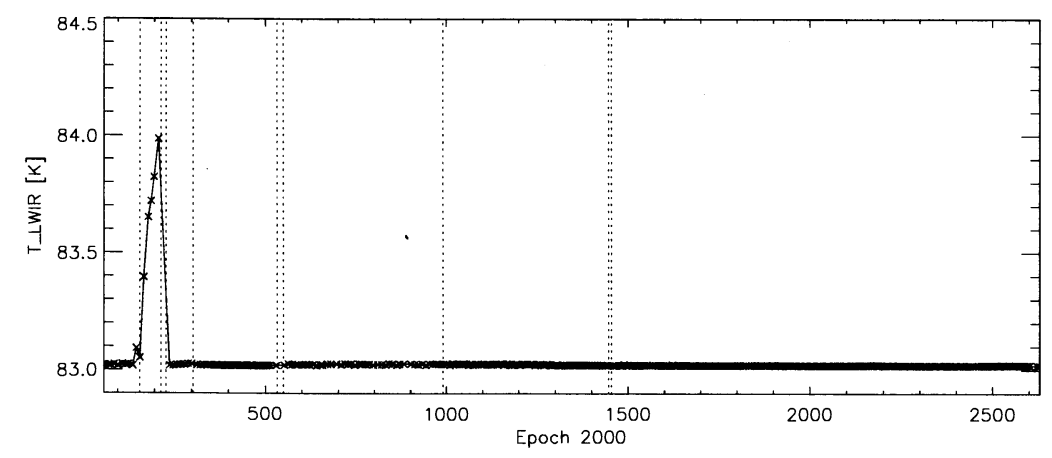


Figure 5
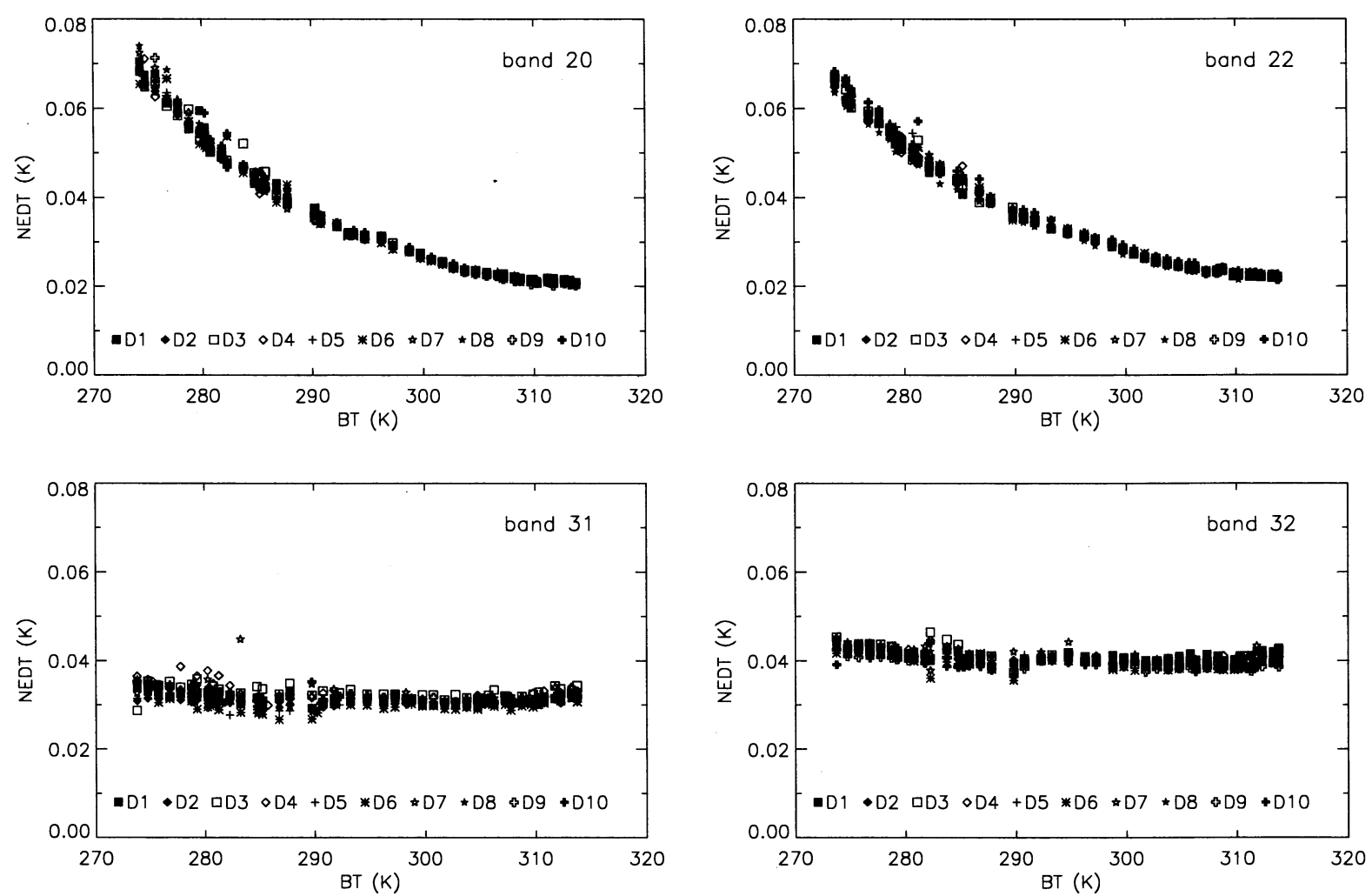
Figure 6
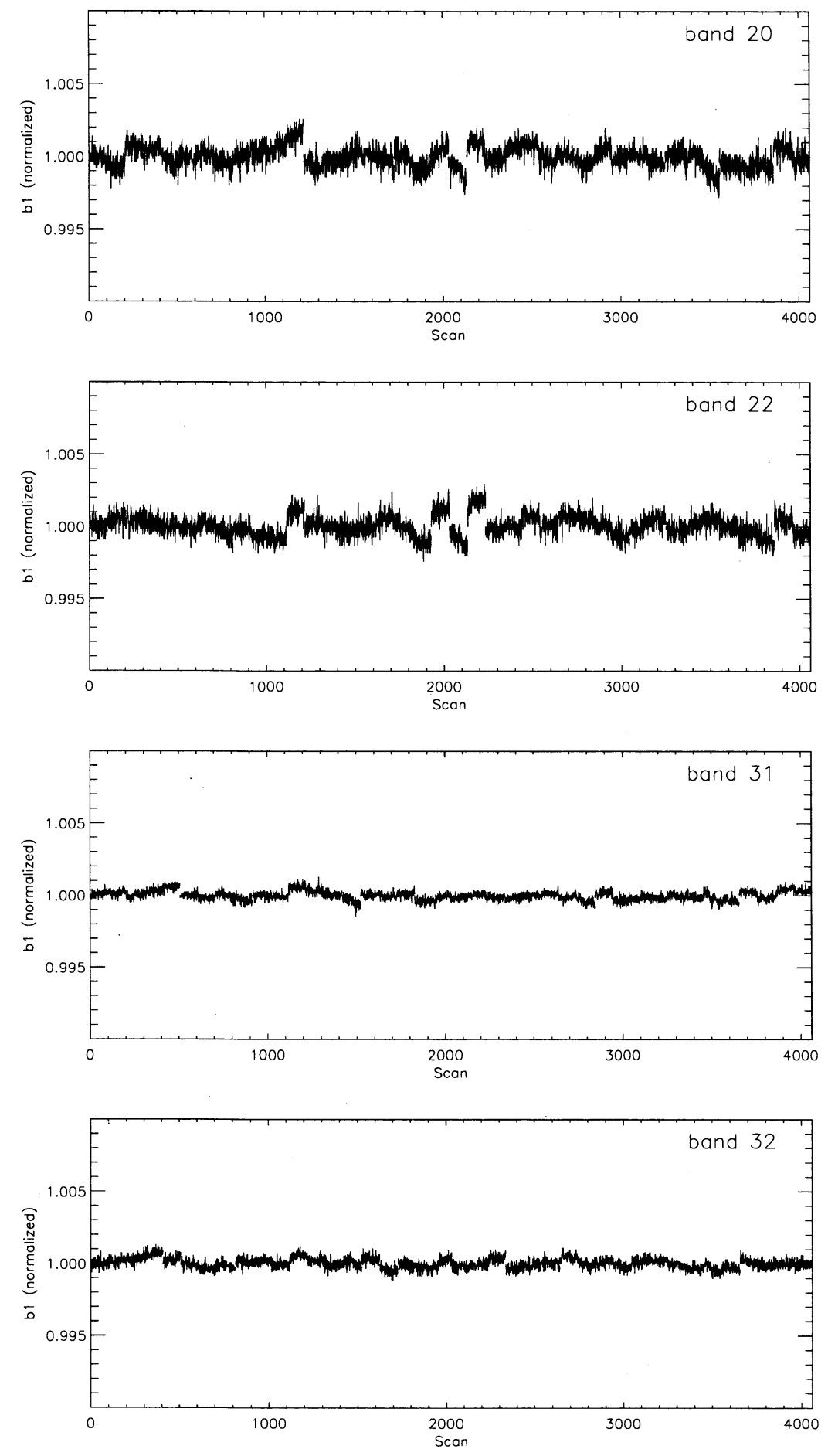
Figure 7

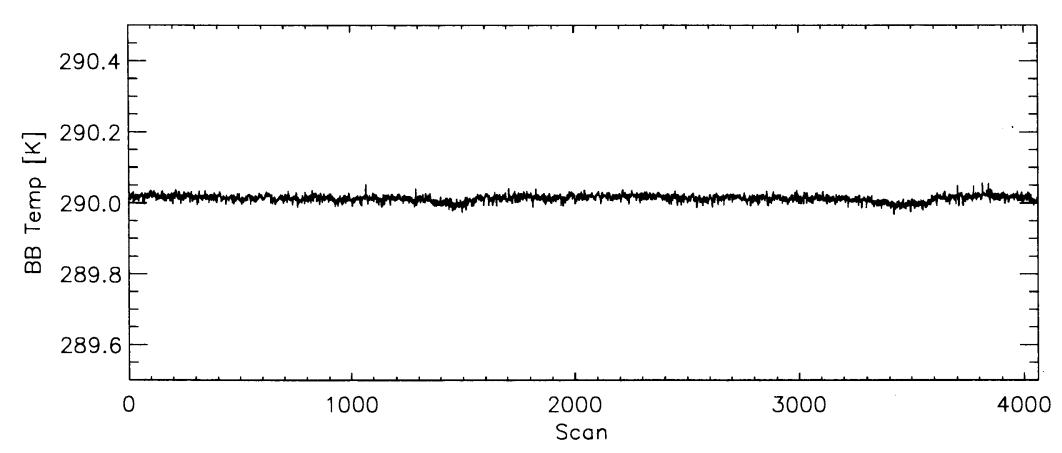

Figure 8

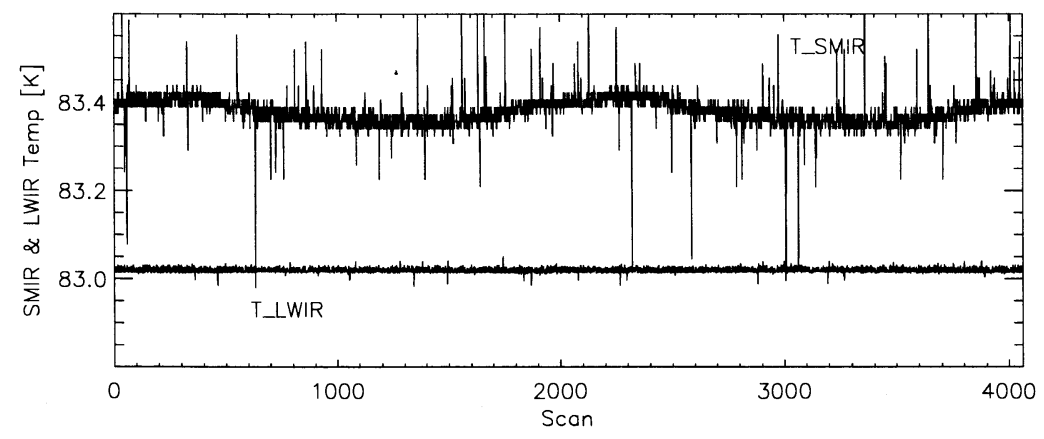


Figure 9
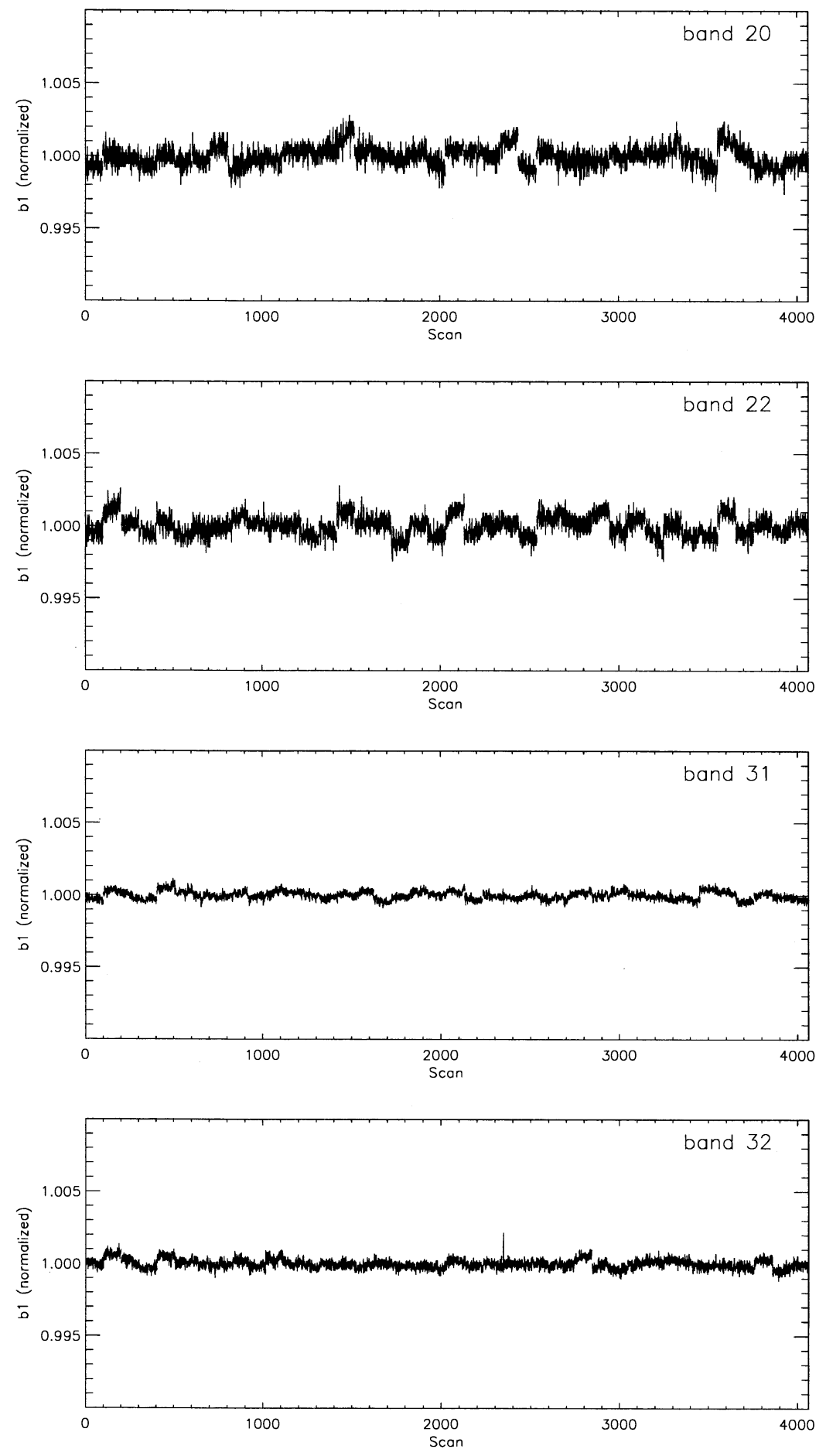
Figure 10

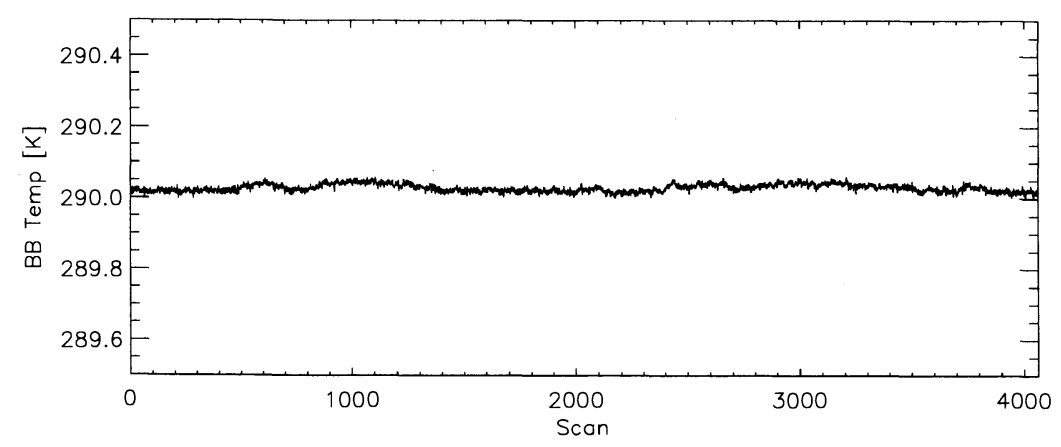

Figure 11

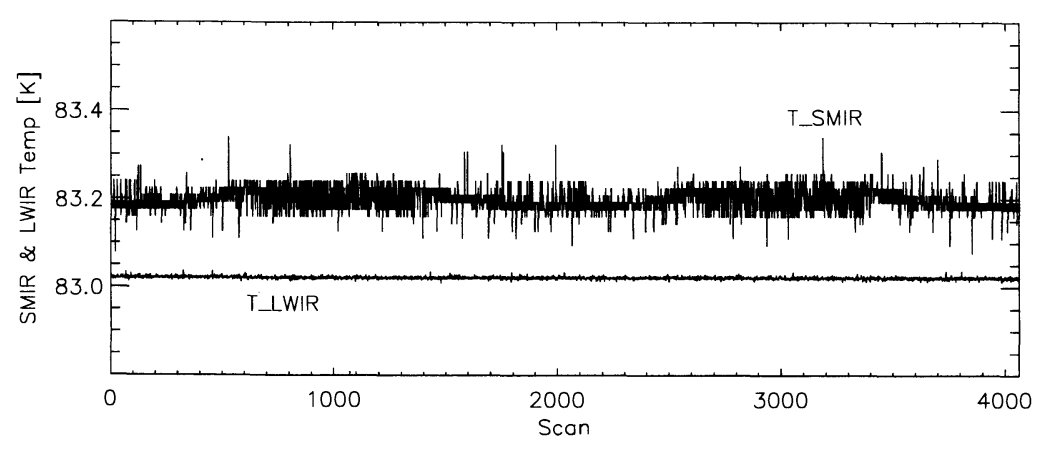


Figure 12
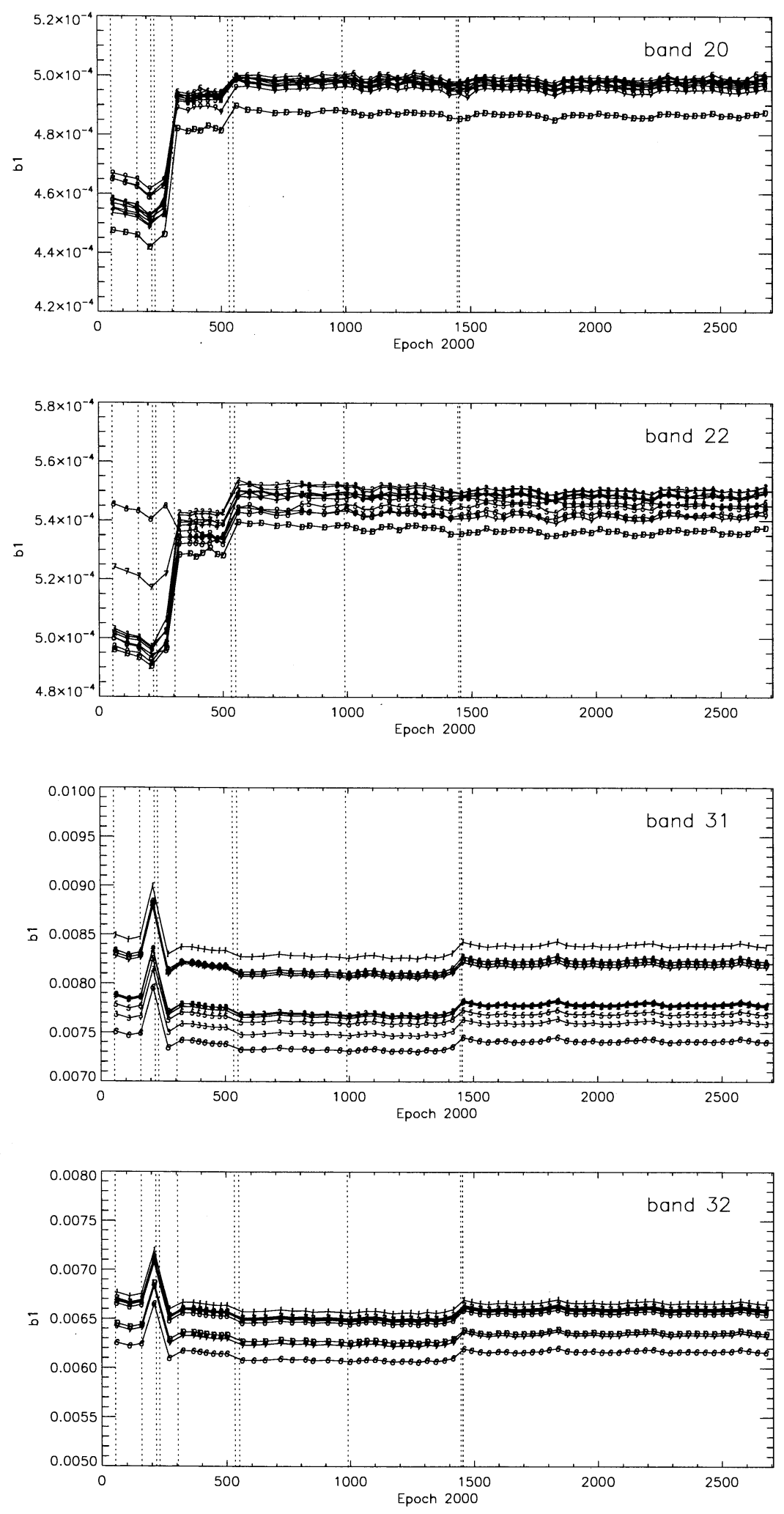
Figure 13
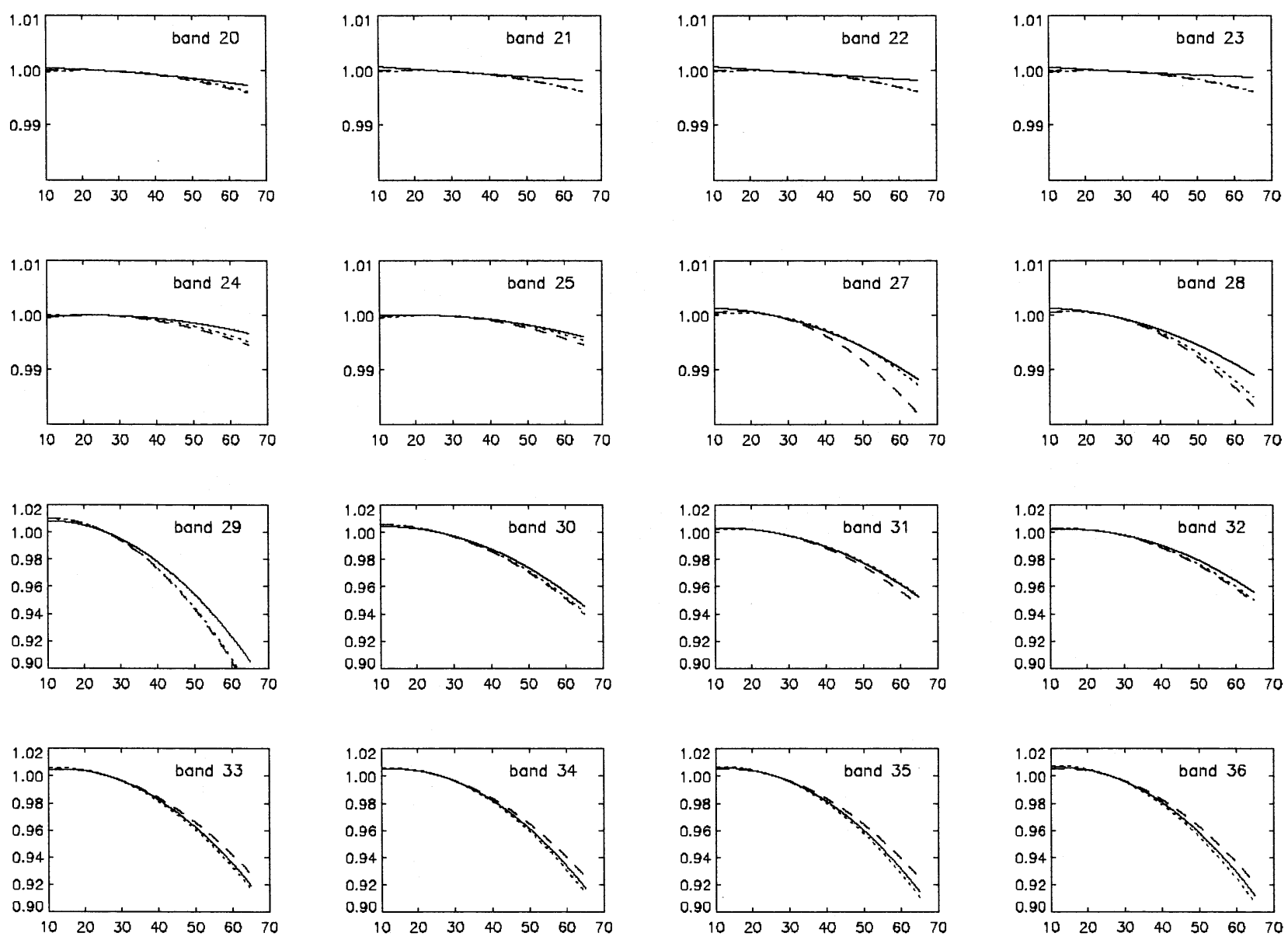
Figure 14
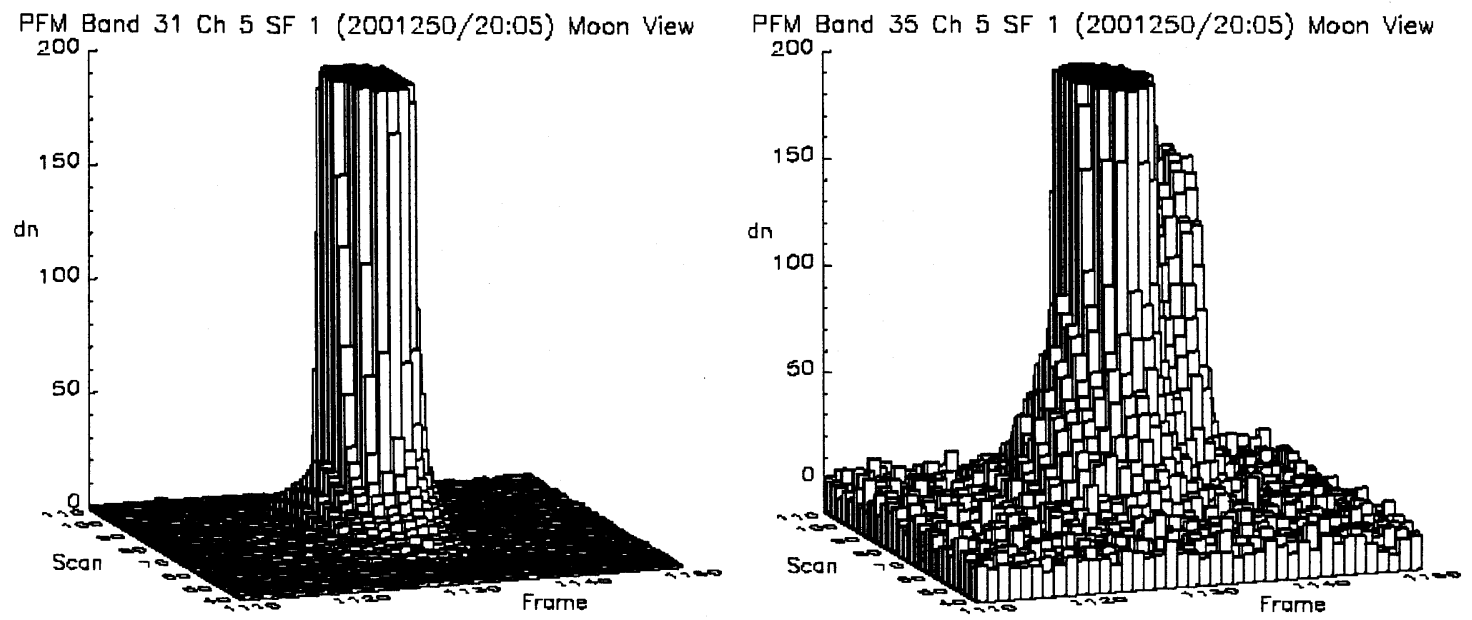

Figure 15
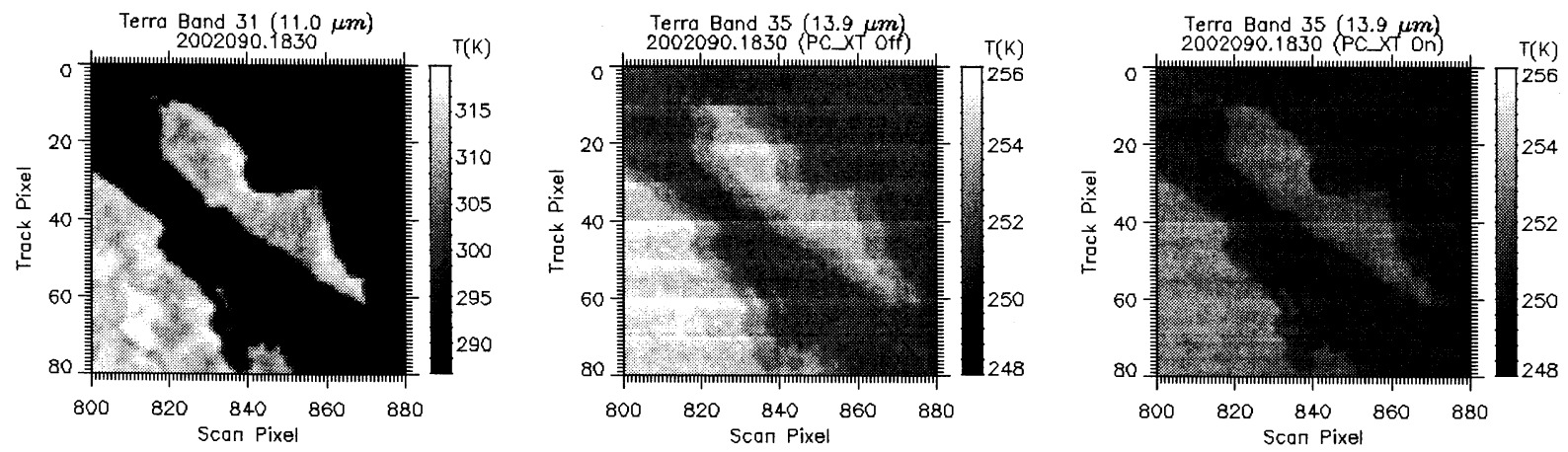

Figure 16
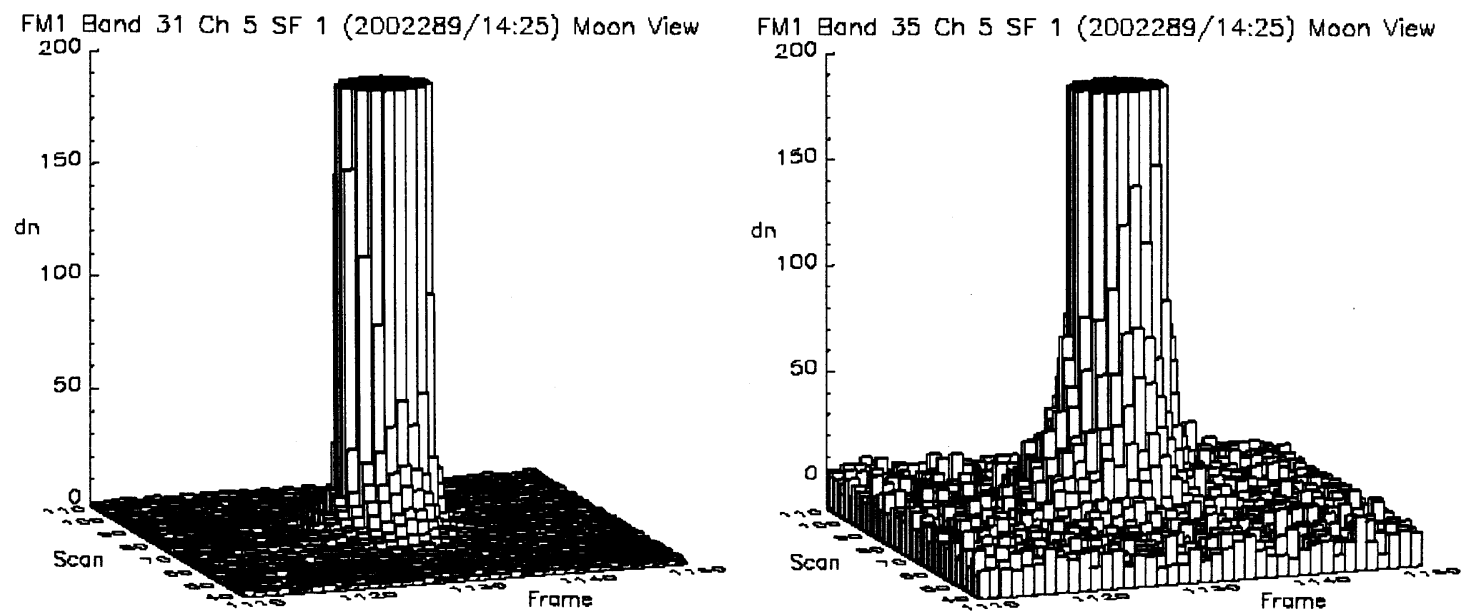